\title{
Density Operators and Quasiprobability Distributions
}

\section{Citation}

Cahill, K. E., and R. J. Glauber. 1969. "Density Operators and Quasiprobability Distributions." Physical Review 177 (5) (January 25): 1882-1902. doi:10.1103/physrev.177.1882.

\section{Published Version}

10.1103/physrev.177.1882

\section{Permanent link}

http://nrs.harvard.edu/urn-3:HUL.InstRepos:33087076

\section{Terms of Use}

This article was downloaded from Harvard University's DASH repository, and is made available under the terms and conditions applicable to Other Posted Material, as set forth at http:// nrs.harvard.edu/urn-3:HUL.InstRepos:dash.current.terms-of-use\#LAA

\section{Share Your Story}

The Harvard community has made this article openly available.

Please share how this access benefits you. Submit a story.

Accessibility 


\title{
Density Operators and Quasiprobability Distributions*
}

\author{
K. E. Cahill $\dagger$ and R. J. Glauber \\ Lyman Laboratory of Physics, Harvard University, Cambridge, Massachusetts 02138
}

(Received 8 July 1968)

\begin{abstract}
The problem of expanding a density operator $\rho$ in forms that simplify the evaluation of important classes of quantum-mechanical expectation values is studied. The weight function $P(\alpha)$ of the $P$ representation, the Wigner distribution $W(\alpha)$, and the function $\langle\alpha|\rho| \alpha\rangle$, where $|\alpha\rangle$ is a coherent state, are discussed from a unified point of view. Each of these quasiprobability distributions is examined as the expectation value of a Hermitian operator, as the weight function of an integral representation for the density operator and as the function associated with the density operator by one of the operator-function correspondences defined in the preceding paper. The weight function $P(\alpha)$ of the $P$ representation is shown to be the expectation value of a Hermitian operator all of whose eigenvalues are infinite. The existence of the function $P(\alpha)$ as an infinitely differentiable function is found to be equivalent to the existence of a well-defined antinormally ordered series expansion for the density operator in powers of the annihilation and creation operators $a$ and $a^{\dagger}$. The Wigner distribution $W(\alpha)$ is shown to be a continuous, uniformly bounded, square-integrable weight function for an integral expansion of the density operator and to be the function associated with the symmetrically ordered power-series expansion of the density operator. The function $\langle\alpha|\rho| \alpha\rangle$, which is infinitely differentiable, corresponds to the normally ordered form of the density operator. Its use as a weight function in an integral expansion of the density operator is shown to involve singularities that are closely related to those which occur in the $P$ representation. A parametrized integral expansion of the density operator is introduced in which the weight function $W(\alpha, s)$ may be identified with the weight function $P(\alpha)$ of the $P$ representation, with the Wigner distribution $W(\alpha)$, and with the function $\langle\alpha|\rho| \alpha\rangle$ when the order parameter $s$ assumes the values $s=+1,0,-1$, respectively. The function $W(\alpha, s)$ is shown to be the expectation value of the ordered operator analog of the $\delta$ function defined in the preceding paper. This operator is in the trace class for $\operatorname{Re} s<0$, has bounded eigenvalues for Res $=0$, and has infinite eigenvalues for $s=1$. Marked changes in the properties of the quasiprobability distribution $W(\alpha, s)$ are exhibited as the order parameter $s$ is varied continuously from $s=-1$, corresponding to the function $\langle\alpha|\rho| \alpha\rangle$, to $s=+1$, corresponding to the function $P(\alpha)$. Methods for constructing these functions and for using them to compute expectation values are presented and illustrated with several examples. One of these examples leads to a physical characterization of the density operators for which the $P$ representation is appropriate.
\end{abstract}

\section{INTRODUCTION}

$T$ HE statistical description of a microscopic system may usually be formulated in terms of its density operator $\rho$. The familiar expression for the statistical average of measurements of a microscopic observable $F$ is the trace of the product of the operators $\rho$ and $F$,

$$
\langle F\rangle=\operatorname{Tr}(\rho F) .
$$

In this paper we discuss ways of writing this statistical average for certain physically important classes of operators $F$ as integrals similar to the phase-space integrals of classical probability theory. The preceding paper $^{1}$ on integral representations for operators and on correspondences between operators and $c$-number functions provides the framework for our analysis ${ }^{2}$ of this problem.

For simplicity we consider only systems which have a single degree of freedom. Further, since we have in mind applications to quantum optics, we describe these systems in terms of the complex operators $a$ and $a^{\dagger}$,

* Supported in part by the National Bureau of Standards and by the Air Force Office of Scientific Research.

$\dagger$ Presently, National Research Council Postdoctoral Research Associate at the National Bureau of Standards, Washington, D. C.

${ }^{1} \mathrm{~K}$. E. Cahill and R. J. Glauber, preceding paper, Phys. Rev. 176, 1857 (1968), hereafter referred to as I; equation numbers cited from it will be prefixed by $\mathrm{I}$.

${ }^{2}$ K. E. Cahill, thesis, Harvard University, 1967, University Microfilms, Ann Arbor (unpublished). Many of the results of the present paper are presented there. which satisfy the commutation relation $\left[a, a^{\dagger}\right]=1$, rather than in terms of their real and imaginary parts $q$ and $p$, for which $[q, p]=i \hbar$. Our discussion is thus directly applicable to a single mode of the electromagnetic field.

A number of procedures for simplifying the evaluation of certain classes of expectation values have been put forward, the first of them by Wigner ${ }^{3}$ and by Moyal. ${ }^{4} \mathrm{~A}$ common feature of these methods is the transfer of statistical information from the density operator $\rho$ to a weight function $w(\alpha)$ which refers to the density operator and whose complex argument $\alpha$ represents a point $\left(q^{\prime}, p^{\prime}\right)$ in the phase space of the system. An expectation value $\langle F\rangle$ is then written as an integral of the product of the weight function $w(\alpha)$ and a function $f(\alpha)$ which refers to the operator $F$,

$$
\operatorname{Tr}(\rho F)=\int w(\alpha) f(\alpha) d^{2} \alpha .
$$

The integration is carried out over all possible states of the system, i.e., over the complex $\alpha$ plane, and the differential $d^{2} \alpha$ is a real element of area proportional to the phase-space element $d q^{\prime} d p^{\prime}$. The function $w(\alpha)$ is not, in general, interpretable as a probability distri-

\footnotetext{
${ }^{3}$ E. P. Wigner, Phys. Rev. 40, 749 (1932).
}

4 J. E. Moyal, Proc. Cambridge Phil. Soc. 45, 99 (1948); 45, 545 (1949).

$177 \quad 1882$ 
bution, but it plays so closely the role of one that we refer to it as a quasiprobability distribution.

The various procedures for expressing expectation values in the form of Eq. (1.2) differ principally in the way in which the functions $w(\alpha)$ and $f(\alpha)$ correspond to the operators $\rho$ and $F$. Because quantum-mechanical operators do not, in general, commute, there are many ways of associating operators with functions. With the function $|\alpha|^{2}$, for example, we could associate either the normally ordered operator $a^{\dagger} a$ or the antinormally ordered operator $a a^{\dagger}$ or, as well, the symmetrized product $\frac{1}{2}\left(a^{\dagger} a+a a^{\dagger}\right)$. The most useful correspondences are based upon these three types of ordering.

In I we have analyzed various ways of defining correspondences between operators and functions. We summarize these results together with some related ones on series and integral expansions for operators in Sec. II.

In Sec. III we discuss a procedure for simplifying expectation values that is based upon the $P$ representation $^{5-15}$ for the density operator. In this representation the density operator assumes the form of a weighted integral over the projection operators upon the coherent states. We show that the weight function $P(\alpha)$ of the $P$ representation is related to the density operator by a correspondence defined in terms of antinormal ordering. ${ }^{2,14}$ The $P$ representation is particularly convenient for dealing with operators that are written in normal order; it affords for the expectation values of the normally ordered products $\left(a^{\dagger}\right)^{n} a^{m}$ the simple integral expressions

$$
\operatorname{Tr}\left[\rho\left(a^{\dagger}\right)^{n} a^{m}\right]=\int P(\alpha)\left(\alpha^{*}\right)^{n} \alpha^{m} d^{2} \alpha .
$$

This relation is a special case of Eq. (1.2) in which the function $w(\alpha)$ associated with the density operator is the weight function $P(\alpha)$ and in which the correspondence between the operator $F$ and the function $f(\alpha)$ is based upon normal ordering.

When the correspondences that associate the functions $w(\alpha)$ and $f(\alpha)$ with the operators $\rho$ and $F$ are both defined in terms of symmetric ordering, the integral (1.2) may be identified with the procedure introduced

\footnotetext{
5 R. J. Glauber, Phys. Rev. Letters 10, 84 (1963).

6 R. J. Glauber, Phys. Rev. 131, 2766 (1963).

7 E. C. G. Sudarshan, Phys. Rev. Letters 10, 277 (1963)

${ }^{8}$ R. J. Glauber, Quantum Optics and Electronics, edited by C. de Witt et al. (Gordon and Breach Science Publishers, Inc., New York, 1965), p. 63.

${ }^{2}$ C. L. Mehta and E. C. G. Sudarshan, Phys. Rev. 138, B274 (1965).

${ }^{10}$ K. E. Cahill, Phys. Rev. 138, B1566 (1965)

11 J. R. Klauder, Phys. Rev. Letters 15, 534 (1966).

12 R. Bonifacio, L. M. Narducci, and E. Montaldi, Phys. Rev. Letters 16, 1125 (1966).

${ }^{13}$ R. Bonifacio, L. M. Narducci, and E. Montaldi, Nuovo Cimento 47, 890 (1967).

${ }_{14}$ M. Lax and W. H. Louisell, J. Quantum Electron. QE3, 47 (1967).

${ }^{16}$ M. M. Miller and E. A. Mishkin, Phys. Rev. 164, 1610 (1967).
}

by Wigner ${ }^{3}$ and by Moyal. ${ }^{4}$ We discuss this procedure in Sec. IV. We show that the Wigner distribution $W(\alpha)$ affords for the density operator an integral representation ${ }^{2,16,17}$ that is considerably more regular than the $P$ representation. In particular, we show that the Wigner distribution is continuous and bounded, being the expectation value of a Hermitian operator whose eigenvalues are \pm 2 . The weight function $P(\alpha)$, by contrast, may be exceedingly singular, being the expectation value of a Hermitian operator all of whose eigenvalues are infinite.

In Sec. $\mathrm{V}$ we discuss a procedure $\mathrm{e}^{8,9,18}$ for dealing with operators written in antinormal order. In this procedure the function corresponding to the weight function $w(\alpha)$ in Eq. (1.2) is the function $\langle\alpha|\rho| \alpha\rangle$, where $|\alpha\rangle$ is a coherent state.

In Sec. VI we unify and relate the procedures discussed in Secs. III-V by using the parametrized ordering convention introduced in $\mathrm{I}$. We construct a parametrized representation for the density operator in which the weight function $W(\alpha, s)$ may be identified with the function $\langle\alpha|\rho| \alpha\rangle$, with the Wigner distribution $W(\alpha)$ and with the weight function $P(\alpha)$ when the order parameter $s$ assumes the values $s=-1,0,+1$, respectively. By varying the order parameter $s$ in a continuous way from $s=-1$ to $s=+1$, we observe an orderly progression of changes in the mathematical properties of this quasiprobability distribution. In this way we are able to see when and how singularities appear in the function $W(\alpha, s)$ and are thereby in a better position to understand, in particular, why the $P$ representation does not exist for important classes of density operators.

We illustrate in Sec. VII the results of the earlier sections by means of several examples which are worked out in detail. We use one of these examples to develop a physical characterization of those density operators for which the $P$ representation is appropriate.

In Sec. VIII we show that the quasiprobability distribution $W(\alpha, s)$ satisfies a partial differential equation similar in form to the heat-diffusion equation. This fact leads to an instructive analogy between the function $W(\alpha, s)$ and a temperature distribution on an infinite plane. Finding the function $W(\alpha, s)$ in terms of the function $W(\alpha, t)$ for $\operatorname{Res}<\operatorname{Re} t$ corresponds to Poisson's solution for the heat-diffusion equation. The inverse problem, of extending the function $W(\alpha, s)$ in the other direction, corresponds to extending a temperature distribution backwards in time, and this problem is solved in Sec. IX.

In Sec. $X$ we return to Eq. (1.2) and discuss the extent to which any procedure for expressing expectation values in that form must resemble the general procedure discussed in Sec. VI.

\footnotetext{
16 U. Fano, Rev. Mod. Phys. 29, 74 (1957).

17 B. R. Mollow, Phys. Rev. 162, 1256 (1967).

18 Y. Kano, J. Math. Phys. 6, 1913 (1965).
} 


\section{ORDERED OPERATOR EXPANSIONS}

The annihilation and creation operators $a$ and $a^{\dagger}$ may be defined for any system that is described by a single pair of Hermitian observables $q$ and $p$, which are canonically conjugate, $[q, p]=i \hbar$. If we form the complex linear combinations

$$
\begin{aligned}
a & =(2 \hbar)^{-1 / 2}\left(\lambda q+i \lambda^{-1} p\right), \\
a^{\dagger} & =(2 \hbar)^{-1 / 2}\left(\lambda q-i \lambda^{-1} p\right),
\end{aligned}
$$

where $\lambda$ is an arbitrary real parameter, then the operators $a$ and $a^{\dagger}$ obey the familiar commutation relation

$$
\left[a, a^{\dagger}\right]=1 \text {. }
$$

A particularly useful complete set of states is the coherent states $|\alpha\rangle$ which are the eigenstates of the operator $a$, i.e., for each complex number $\alpha$ we have

$$
a|\alpha\rangle=\alpha|\alpha\rangle
$$

The basic properties of the coherent states and of the unitary displacement operators

$$
D(\alpha)=\exp \left(\alpha a^{\dagger}-\alpha^{*} a\right),
$$

which generate them from the state $|0\rangle$ are described in Secs. II and III of I.

The space of eigenvalues or the phase space for our dynamical system is the infinite plane of eigenvalues $\left(q^{\prime}, p^{\prime}\right)$ of the Hermitian observables $q$ and $p$. An equivalent phase space is the complex plane of eigenvalues $^{19} \quad \alpha=(2 \pi h)^{-1}\left(\lambda q^{\prime}+i \lambda^{-1} p^{\prime}\right)$ of the annihilation operator $a$. As in I, we make use, in integrating over the complex plane, of the convenient differential element of area

$$
\pi^{-1} d^{2} \alpha=\pi^{-1} d(\operatorname{Re} \alpha) d(\operatorname{Im} \alpha)=(2 \pi h)^{-1} d q^{\prime} d p^{\prime}
$$

which we see is also the familiar (dimensionless) element of phase space.

In I we examined the problem of representing an arbitrary operator $F$ both as a power series in the operators $a$ and $a^{\dagger}$ and as an integral over various complete and quasicomplete sets of operators. An important device in our treatment was a parametrization of the usual ways of ordering operators according to which normal order, antinormal order, and a type of ordering that is symmetric in the operators $a$ and $a^{\dagger}$ correspond to three discrete values of a continuous order parameter $s$.

We defined the $s$-ordered products $\left\{\left(a^{\dagger}\right)^{n} a^{m}\right\}_{s}$ of the operators $\left(a^{\dagger}\right)^{n}$ and $a^{m}$ by means of a Taylor-series

\footnotetext{
${ }^{19}$ The coherent state $|\alpha\rangle$ is not an eigenstate of either $q$ or $p$. The quantities $q^{\prime}$ and $p^{\prime}$ in this expression may be interpreted as the expectation values of $q$ and $p$ in the state $|\alpha\rangle$.
}

expansion of the operator

$$
\begin{aligned}
D(\alpha, s) & \equiv D(\alpha) e^{s|\alpha|^{2 / 2}} \\
& =\exp \left(\alpha a^{\dagger}-\alpha^{*} a+\frac{1}{2} s|\alpha|^{2}\right) \\
& =\sum_{n, m=0}^{\infty} \frac{\alpha^{n}\left(-\alpha^{*}\right)^{m}}{n ! m !}\left\{\left(a^{\dagger}\right)^{n} a^{m}\right\}_{s} .
\end{aligned}
$$

Since the operators $D(\alpha, 1)$ and $D(\alpha,-1)$ are the normally and antinormally ordered exponentials

$$
\begin{aligned}
D(\alpha, 1) & =\exp \left(\alpha a^{\dagger}\right) \exp \left(-\alpha^{*} a\right), \\
D(\alpha,-1) & =\exp \left(-\alpha^{*} a\right) \exp \left(\alpha a^{\dagger}\right),
\end{aligned}
$$

the normally and antinormally ordered products are distinguished by the values $s= \pm 1$ :

$$
\begin{aligned}
\left\{\left(a^{\dagger}\right)^{n} a^{m}\right\}_{1} & =\left(a^{\dagger}\right)^{n} a^{m}, \\
\left\{\left(a^{\dagger}\right)^{n} a^{m}\right\}_{-1} & =a^{m}\left(a^{\dagger}\right)^{n} .
\end{aligned}
$$

The operator $\left\{\left(a^{\dagger}\right)^{n} a^{m}\right\}_{0}$ corresponding to the value $s=0$, is the average of all ways of ordering the product of $n$ factors of $a^{\dagger}$ and $m$ factors of $a$. We have therefore designated the type of ordering which emerges for $s=0$ as symmetric ordering.

In Secs. IV and V of I, we discussed the problem of expanding an operator $F$ as a power series in the $s$ ordered products, i.e., in the form

$$
F=\sum_{n, m=0}^{\infty} f_{n, m}(s)\left\{\left(a^{\dagger}\right)^{n} a^{m}\right\}_{s}
$$

where the coefficients $f_{n, m}(s)$ are complex numbers. We derived explicit formulas for the coefficients $f_{n, m}(s)$ and defined a type of convergence that is appropriate for the power series (2.12). For the case of normal ordering, $s=1$, we found the expansion (2.12) converges according to this definition for an extremely broad class of operators $F$. For Res $>0$ or, equivalently, for orderings closer to normal order than to antinormal order, we showed that the coefficients $f_{n, m}(s)$ are finite for a more restricted class of operators $F$. These operators, which we refer to as the class of bounded operators, are the ones for which the Hilbert-Schmidt norm

$$
\|F\|=\left[\operatorname{Tr}\left(F^{\dagger} F\right)\right]^{1 / 2}
$$

is finite. For such operators we also showed that the power series (2.12) converges for $\operatorname{Res}>\frac{1}{2}+\frac{1}{2}(\operatorname{Im} s)^{2}$, i.e., for orderings that are closer to normal order than to symmetric order.

For the case of antinormal ordering, $s=-1$, however, and in fact throughout the half-plane $\operatorname{Re} s \leq 0$, the coefficients $f_{n, m}(s)$ can and do develop singularities. These singularities, which raise questions as to the meaning of expansions such as Eq. (2.12), occur even for operators $F$ that are both bounded and in the trace class. Broadly speaking, the more negative the real part of $s$ the more restricted must be our interpretation 
of the expansion (2.12); this limitation is far more restrictive for antinormal order, $s=-1$, than for symmetric order, $s=0$.

In Secs. VI-VIII of I we discussed a class of integral expansions for operators. These expansions are based upon the operators $T(\alpha, s)$ which we defined as the complex Fourier transforms ${ }^{20}$ of the operators $D(\alpha, s)$,

$$
T(\alpha, s)=\int D(\xi, s) \exp \left(\alpha \xi^{*}-\alpha^{*} \xi\right) \pi^{-1} d^{2} \xi
$$

The expansions for an arbitrary operator $F$ take the form

$$
F=\int f(\alpha,-s) T(\alpha, s) \pi^{-1} d^{2} \alpha
$$

in which the weight function $f(\alpha,-s)$ is given by the trace

$$
f(\alpha,-s)=\operatorname{Tr}[F T(\alpha,-s)] \text {. }
$$

An important property of these expansions is the close relationship which exists between the operator $F$ being expanded and its weight function $f(\alpha,-s)$. The powerseries expansion of the weight function $f(\alpha,-s)$ possesses the same coefficients $f_{n, m}(s)$ that occur in the $s$-ordered power-series expansion (2.12) of the operator $F$, i.e., we have

$$
f(\alpha,-s)=\sum_{n, m=0}^{\infty} f_{n, m}(s)\left(\alpha^{*}\right)^{n} \alpha^{m} .
$$

In other words, $f(\alpha,-s)$ is the same function of $\alpha$ and $\alpha^{*}$ as the $s$-ordered version of $F$ is of $a$ and $a^{\dagger}$.

We may regard this identity of the coefficients $f_{n, m}(s)$ in the series (2.12) and (2.17) as defining, for each value of the order parameter $s$, a one-to-one correspondence between operators and functions. We have referred to this correspondence between operators $F$ and their weight functions $f(\alpha,-s)$ as the correspondence $C(s)$. The rule of the correspondence $C(s)$

$$
F \underset{s}{\leftrightarrow} f(\alpha,-s)=\operatorname{Tr}[F T(\alpha,-s)]
$$

is that the $s$-ordered product $\left\{\left(a^{\dagger}\right)^{n} a^{m}\right\}_{s}$ is associated with the monomial $\left(\alpha^{*}\right)^{n} \alpha^{m}$

$$
\left\{\left(a^{\dagger}\right)^{n} a^{m}\right\}_{s} \leftrightarrow\left(\alpha^{*}\right)^{n} \alpha^{m}
$$

As in the case of the power series expansion (2.12), the properties of the integral representation (2.15) and of the weight function $f(\alpha,-s)$ depend critically upon the order parameter $s$. In general for bounded operators $F$ the weight functions $f(\alpha,-s)$ are wellbehaved functions of $\alpha$ for $\operatorname{Res} \geq 0$ but for $\operatorname{Re}<<0$ they can develop singularities. These singularities are closely

${ }^{20}$ This type of Fourier transformation, which differs from the usual one only by a change of scale, is defined by Eq. (I.3.1). related to those that occur in the coefficients $f_{n, m}(s)$ of the power-series expansion (2.12). For according to Eq. (2.17), the weight function $f(\alpha,-s)$ is a generating function for the coefficients $f_{n, m}(s)$. When this function is infinitely differentiable, then we may write the operator $F$ in the form

$$
F=\left.\sum_{n, m=0}^{\infty} \frac{1}{n ! m !}\left\{\left(a^{\dagger}\right)^{n} a^{m}\right\}_{s}\left(\frac{\partial^{n+m} f(\alpha,-s)}{\partial\left(\alpha^{*}\right)^{n} \partial \alpha^{m}}\right)\right|_{\alpha=0},
$$

which is another way of expressing the operatorfunction correspondence $C(s)$.

\section{THE $P$ REPRESENTATION}

The utility of normal ordering has long been recognized. It is used, for example, in the Dyson-Wick expansion of the scattering matrix in quantum electrodynamics. ${ }^{21}$ Because of the appropriateness of normally ordered products for the description of photon absorption processes, normal ordering has had new applications, recently, in the interpretation of photon counting and coherence experiments. ${ }^{6,8,22}$. The correlation functions $G^{(n)}$ for the quantized field are defined, for example, as expectation values of normally. ordered products of the positive-frequency part of the electric field operator $E_{\mu}{ }^{(+)}(x)$ and the negativefrequency part $E_{\mu}^{(-)}(x)$. The $n$ th-order correlation function may be written as

$G^{(n)}\left(x_{1}, x_{2}, \cdots, x_{n}, x_{n+1}, \cdots, x_{2 n}\right)=\operatorname{Tr}\left[\rho E^{(-)}\left(x_{1}\right) \cdots\right.$
$\left.E^{(-)}\left(x_{n}\right) E^{(+)}\left(x_{n+1}\right) \cdots E^{(+)}\left(x_{2 n}\right)\right]$

where we have denoted $2 n$ sets of space-time coordinates as $x_{1} \cdots x_{2 n}$ and have suppressed the vector polarization indices of the field operators.

We have mentioned that the $P$ representation for the density operator,

$$
\rho=\int P(\alpha)|\alpha\rangle\langle\alpha| d^{2} \alpha
$$

affords a convenient way of evaluating the ensemble averages of normally ordered operators. It has apart from this important use a variety of additional applications ${ }^{17,23-26}$ because of the elementary character of the coherent states $|\alpha\rangle$. Among these applications is the particularly simple form which it provides for a quantum-mechanical analog ${ }^{6}$ of the classical superposition principle for the electromagnetic field.

We may use the $P$ representation to write the expectation value of an arbitrary operator $F$ as the

${ }^{21}$ G. C. Wick, Phys. Rev. 80, 268 (1950).

22 R. J. Glauber, Phys. Rev. 130, 2529 (1963).

${ }^{23}$ R. J. Glauber, Phys. Letters 21, 650 (1966).

24 B. R. Mollow and R. J. Glauber, Phys. Rev. 160, 1076 (1967); 160, 1097 (1967).

${ }^{25}$ Y. R. Shen, Phys. Rev. 155, 921 (1967)

${ }^{26}$ R. J. Glauber, in Proceedings of the Physics of Quantum Electronics Conference, San Juan, Puerto Rico, 1965, edited by P. L. Kelly et al. (McGraw-Hill Book Co., New York, 1966), p. 788. 
integral

$$
\begin{aligned}
\operatorname{Tr}(\rho F) & =\int P(\alpha) \operatorname{Tr}[|\alpha\rangle\langle\alpha| F] d^{2} \alpha \\
& =\int P(\alpha)\langle\alpha|F| \alpha\rangle d^{2} \alpha .
\end{aligned}
$$

The association of the function

$$
f(\alpha,-1)=\langle\alpha|F| \alpha\rangle
$$

with the operator $F$ defines a particularly simple correspondence between operators and functions.

Let us suppose that the operator $F$ possesses a normally ordered power series of the form

$$
F=\sum_{n, m=0}^{\infty} f_{n, m}(1)\left(a^{\dagger}\right)^{n} a^{m} .
$$

As we showed in I, such series exist and converge for an extremely broad class of operators $F$, including all that satisfy the condition (I.4.23). Now because of the eigenvalue property of the coherent states, the function $f(\alpha)=\langle\alpha|F| \alpha\rangle$ may be obtained from the operator $F$ by replacing the operators $a$ and $a^{\dagger}$ in the series (3.5) by their complex eigenvalues $\alpha$ and $\alpha^{*}$, so that we have

$$
f(\alpha,-1)=\sum_{n, m=0}^{\infty} f_{n, m}(1)\left(\alpha^{*}\right)^{n} \alpha^{m} .
$$

It is this function which occurs in the integral (3.3) for the ensemble average of the operator $F$,

$$
\operatorname{Tr}(\rho F)=\int P(\alpha) f(\alpha,-1) d^{2} \alpha .
$$

Since the series (3.5) and (3.6), as was shown in I, converge for virtually all operators of interest, this expression is completely reliable provided that the $P$ representation exists for the density operator $\rho$.

We observe that, since all density operators are Hermitian and of unit trace, the weight function $P(\alpha)$, when it exists, can be chosen to be real and normalized to unity,

$$
1=\operatorname{Tr} \rho=\int P(\alpha) d^{2} \alpha
$$

Having defined the $P$ representation and touched upon its usefulness, we shall now consider it from a variety of viewpoints each of which reveals a way of constructing the weight function $P(\alpha)$. All of these ways show a certain lack of generality which reflects the fact that the $P$ representation does not exist for all density operators.

Let us note that by using the resolution of the identity operator in terms of the coherent states, Eq. (I.2.27), we may write the antinormally ordered products $a^{m}\left(a^{\dagger}\right)^{n}$ in the form

$$
\begin{aligned}
a^{m}\left(a^{\dagger}\right)^{n} & =a^{m} \int|\alpha\rangle\langle\alpha| \pi^{-1} d^{2} \alpha\left(a^{\dagger}\right)^{n} \\
& =\int\left(\alpha^{*}\right)^{n} \alpha^{m}|\alpha\rangle\langle\alpha| \pi^{-1} d^{2} \alpha .
\end{aligned}
$$

Let us now suppose that the density operator $\rho$ possesses the antinormally ordered power series

$$
\rho=\sum_{n, m=0}^{\infty} \rho_{n, m}(-1) a^{m}\left(a^{\dagger}\right)^{n} .
$$

It then follows from Eq. (3.9) that this density operator also possesses the $P$ representation (3.2) with the function

$$
P(\alpha)=\pi^{-1} \sum_{n, m=0}^{\infty} \rho_{n, m}(-1)\left(\alpha^{*}\right)^{n} \alpha^{m}
$$

as its weight function. ${ }^{2,14}$ The function $P(\alpha)$ is thus a generating function for the coefficients of the antinormally ordered power-series expansion (3.10) of the density operator $\rho$. Equivalently, we may say that the correspondence that associates a density operator $\rho$ with its weight function $P(\alpha)$ is the one based upon antinormal ordering, $s=-1$.

This correspondence does not, however, constitute a general prescription for constructing the weight function $P(\alpha)$. For, as we have seen in Secs. IV and V of I, the coefficients $f_{n, m}(-1)$ of antinormally ordered power-series expansions are sometimes singular, even for operators that are both bounded and in the trace class.

As another approach to finding the function $P(\alpha)$ let us note that the function $f(\alpha,-s)$ associated with an arbitrary operator $F$ by the correspondence $C(s)$ based upon s-ordering is in general given by Eq. (2.18) as the trace

$$
f(\alpha,-s)=\operatorname{Tr}[F T(\alpha,-s)] .
$$

Since all density operators are both bounded and in the trace class, there is no intrinsic difficulty in applying this relation to an arbitrary density operator, $F=\rho$. If, therefore, we set $s=-1$, corresponding to antinormal ordering, then we may write the weight function $P(\alpha)$ as the trace

$$
P(\alpha)=\pi^{-1} \operatorname{Tr}[\rho T(\alpha, 1)] .
$$

This trace is simply the expectation value of the operator $T(\alpha, 1)$.

Let us recall that the operator $T(\alpha, s)$, defined by Eq. (2.14), is Hermitian for real values of $s$, as shown by Eq. (I.6.17) and is in the trace class for Res $<0$, as shown by Eq. (I.6.32). According to Eq. (I.6.28), the eigenvalues $e_{n}(s)$ of the operator $T(\alpha, s)$ are independent 
of $\alpha$ and are given by

$$
e_{n}(s)=\frac{2}{1-s}\left(\frac{s+1}{s-1}\right)^{n} \text {. }
$$

We see that they are all infinite for $s=1$.

The fact that the function $P(\alpha)$ is the expectation value of a Hermitian operator $T(\alpha, 1)$ all of whose eigenvalues $e_{n}(1)$ are infinite is related to the occurrence of singularities in the coefficients $\rho_{n, m}(-1)$ of the antinormally ordered power series (3.10) for the density operator $\rho$. Furthermore, it sheds some light on why the weight function $P(\alpha)$ is, for certain classes of density operators, exceedingly singular. ${ }^{10}$

We shall provide a fuller discussion of these singularities at the end of this section and also in Secs. VI and VII in which we make use of the continuous order parameter $s$. For the present let us merely note that Eq. (3.13) is not well defined as it stands. It may be made meaningful for a broad class of density operators $\rho$ if we use a limiting process in which the order parameter $s$ approaches one along the real axis from smaller values, i.e.,

$$
P(\alpha)=\pi^{-1} \lim _{s \rightarrow 1-} \operatorname{Tr}[\rho T(\alpha, s)] .
$$

Let us recall that, according to Eq. (I.6.29), the operator $T(\alpha, s)$ is for $s=-1$ simply the projection operator upon the coherent state $|\alpha\rangle$,

$$
T(\alpha,-1)=|\alpha\rangle\langle\alpha|
$$

We may thus write the $P$ representation in the form

$$
\rho=\int P(\alpha) T(\alpha,-1) d^{2} \alpha .
$$

We note that this expansion conforms to Eqs. (2.15) and (2.16) when the weight function $P(\alpha)$ is given by Eq. (3.13). If we now multiply both sides of the expansion (3.17) by an arbitrary operator $F$ and form the trace of the resulting expression, then we arrive at the relation

$$
\begin{aligned}
& \operatorname{Tr}(\rho F) \\
& =\int \operatorname{Tr}[\rho T(\alpha, 1)] \operatorname{Tr}[F T(\alpha,-1)] \pi^{-1} d^{2} \alpha,
\end{aligned}
$$

where we have used Eq. (3.13) for $P(\alpha)$. In this integral for the quantity $\operatorname{Tr}(\rho F)$ the order parameter $s$ occurs twice, as $s=+1$ and as $s=-1$, in accordance with the occurrence of both $s$ and $-s$ in the general relation (2.15).

We shall now derive what is perhaps the simplest way of constructing the weight function $P(\alpha)$ when it is nonsingular. By substituting our definition (2.14) of the operator $T(\alpha, s)$ into Eq. (3.15), we may express the function $P(\alpha)$ in terms of the limiting process

$P(\alpha)=\pi^{-2} \lim _{s \rightarrow 1-} \int \exp \left(\alpha \xi^{*}-\alpha^{*} \xi\right) \operatorname{Tr}[\rho D(\xi, s)] d^{2} \xi$

Let us now define the normally ordered characteristic function $\chi_{N}(\xi)$ as the expectation value ${ }^{26}$

$$
\begin{aligned}
\chi_{N}(\xi) & =\operatorname{Tr}[\rho D(\xi, 1)] \\
& =\operatorname{Tr}\left[\rho \exp \left(\xi a^{\dagger}\right) \exp \left(-\xi^{*} a\right)\right] .
\end{aligned}
$$

For many density operators $\rho$ the function $\chi_{N}(\xi)$ possesses a complex Fourier transform ${ }^{20}$; and, when that is the case, the limiting process (3.19) is unnecessary. For these density operators the weight function $P(\alpha)$ is the complex Fourier transform of the normally ordered characteristic function ${ }^{26}$

$$
P(\alpha)=\pi^{-2} \int \exp \left(\alpha \xi^{*}-\alpha^{*} \xi\right) \chi_{N}(\xi) d^{2} \xi
$$

and by using Eqs. (I.3.4) and (I.3.5) we find as the inverse relation

$$
\chi_{N}(\xi)=\int \exp \left(\xi \alpha^{*}-\xi^{*} \alpha\right) P(\alpha) d^{2} \alpha .
$$

For other density operators, the limiting process (3.19) typically leads to a generalized function that is too singular to be used as a weight function for the $P$ representation. ${ }^{27}$ For the case of pure states, for example, it has been shown ${ }^{28}$ that the function $P(\alpha)$ is always singular and that it is a tempered distribution only when the state may be expressed as a linear combination of a finite number of the states $\left(a^{\dagger}\right)^{n}|\alpha\rangle$ for a single, arbitrary value of $\alpha$.

We have seen that the $P$ representation provides for the ensemble averages of the normally ordered products $\left(a^{\dagger}\right)^{n} a^{m}$ the simple expression (1.3). Let us now observe that by using Eq. (I.5.14) for example we may use the $P$ representation to write the ensemble averages of the symmetrically and antinormally ordered products in the forms

$$
\begin{array}{r}
\operatorname{Tr}\left[\rho\left\{\left(a^{\dagger}\right)^{n} a^{m}\right\}_{0}\right]=n ! 2^{-n} \int \alpha^{m-n} L_{n}{ }^{(m-n)}\left(-2|\alpha|^{2}\right) \\
\\
\times P(\alpha) d^{2} \alpha
\end{array}
$$

${ }^{27} \mathrm{~K}$. E. Cahill (to be published). It is shown there that it is possible to regularize the $P$ representation so as to accommodate such generalized functions but only at the expense of adding to it three two-dimensional integrals over outer products of nonidentical coherent states. This regularized $P$ representation exists with four nonsingular weight functions for all density operators $\rho$. Only when the three supplementary weight functions can be set equal to zero, however, does the $P$ representation exist.

${ }^{28} \mathrm{~K}$. E. Cahill (to be published). 
and

$$
\begin{array}{r}
\operatorname{Tr}\left[\rho a^{m}\left(a^{\dagger}\right)^{n}\right]=n ! \int \alpha^{m-n} L_{n}{ }^{(m-n)}\left(-|\alpha|^{2}\right) \\
\times P(\alpha) d^{2} \alpha,
\end{array}
$$

where $L_{n}{ }^{(m)}(x)$ is an associated Laguerre polynomial. ${ }^{29}$

\section{WIGNER DISTRIBUTION}

In this section we discuss a representation for the density operator that is particularly suited for the averaging of operators written in symmetric order. This representation exists for all density operators and affords a way of expressing the expectation value of every bounded operator as a convergent integral. The weight function of this representation is the quasiprobability distribution function introduced by Wigner. ${ }^{3}$ Our discussion will begin with the displacement-operator expansion in which operators are expressed in a form that is implicitly symmetric in the operators $a$ and $a^{\dagger}$.

We have seen in Sec. III of I that the displacement operators $D(\alpha)$ form a complete set of operators. They afford for every operator $F$ that is bounded in the sense of Eq. (2.13) an expansion of the form

$$
F=\int f(\xi) D^{-1}(\xi) \pi^{-1} d^{2} \xi
$$

in which the weight function

$$
f(\xi)=\operatorname{Tr}[F D(\xi)]
$$

is unique and square-integrable. Thus since every density operator is bounded, we may write an arbitrary density operator in the form

$$
\rho=\int \chi(\xi) D^{-1}(\xi) \pi^{-1} d^{2} \xi
$$

where the weight function $\chi(\xi)$ is given by the trace

$$
\chi(\xi)=\operatorname{Tr}[\rho D(\xi)]
$$

which is the expectation value of the displacement operator $D(\xi)$. This relation is the familiar definition of the characteristic function, as contrasted with the normally ordered form considered in Sec. III. It plays a role in quantum-statistical theory analogous to that of the characteristic function of classical probability theory. According to Eq. (I.3.14) we have

$$
\int|\chi(\xi)|{ }^{2} \pi^{-1} d^{2} \xi=\operatorname{Tr} \rho^{2} \leq 1,
$$

which shows that the characteristic function $\chi(\xi)$ is always square-integrable. It is clear from the expansion

\footnotetext{
${ }^{29} \mathrm{~W}$. Magnus and F. Oberhettinger, Formulas and Theorems for the Functions of Mathematical Physics (Chelsea Publishing Co., New York, 1954), p. 85.
}

(4.3), that the function $\chi(\xi)$ uniquely determines the density operator $\rho$.

Since the displacement operator $D(\xi)$ is unitary for all $\xi$ and unity for $\xi=0$, the characteristic function $\chi(\xi)$, which is its expectation value, must satisfy the conditions

$$
|\chi(\xi)| \leq 1, \quad \chi(0)=1 .
$$

The boundedness condition on $\chi(\xi)$ contrasts sharply with the growth condition that applies to the normally ordered characteristic function $\chi_{N}(\xi)$. If we compare the definitions (3.20) and (4.4) with Eq. (I.2.14), then we may deduce from the condition (4.6) that the function $\chi_{N}(\xi)$ must obey the growth condition

$$
\left|\chi_{N}(\xi)\right| \leq e^{|\xi|^{2 / 2}} .
$$

The possibility of such exponential growth at large values of $|\xi|$ is the reason why the function $\chi_{N}(\xi)$ does not naturally possess a Fourier transform, even one that is a tempered distribution.

In the case of the characteristic function $\chi(\xi)$, however, which is both bounded and square-integrable, we encounter no difficulty in defining its complex Fourier transform as

$$
W(\alpha)=\int \exp \left(\alpha \xi^{*}-\alpha^{*} \xi\right) \chi(\xi) \pi^{-1} d^{2} \xi .
$$

This function differs only in normalization from the distribution $W\left(q^{\prime}, p^{\prime}\right)$ introduced by Wigner $^{3}$ as a quantum-mechanical analog of the phase-space distribution of classical statistical mechanics. By using the Fourier inversion relations (I.3.4) and (I.3.5), we may express the function $\chi(\xi)$ in terms of the Wigner function as

$$
\chi(\xi)=\int \exp \left(\xi \alpha^{*}-\xi^{*} \alpha\right) W(\alpha) \pi^{-1} d^{2} \alpha .
$$

If we now substitute this expression for the characteristic function into the expansion (4.3) and use the definition (2.14) of the operator $T(\alpha, 0)$, we arrive at the representation ${ }^{2,16,17}$

$$
\rho=\int W(\alpha) T(\alpha, 0) \pi^{-1} d^{2} \alpha .
$$

The Wigner function $W(\alpha)$ is therefore a weight function for the expansion of the density operator in terms of the operators $T(\alpha, 0)$ which, according to Eq. (I.6.23), are given by

$$
T(\alpha, 0)=2 D(\alpha)(-1)^{a \dagger a} D^{-1}(\alpha) .
$$

By writing Eq. (4.8) in the form

$$
W(\alpha)=\operatorname{Tr}\left[\rho \int \exp \left(\alpha \xi^{*}-\alpha \xi^{*}\right) D(\xi) \pi^{-1} d^{2} \xi\right],
$$


and using the definition (2.14), we find that the Wigner distribution is the expectation value of the operator $T(\alpha, 0)$ :

$$
W(\alpha)=\operatorname{Tr}[\rho T(\alpha, 0)] .
$$

Since the operator $T(\alpha, 0)$ is Hermitian, as shown by Eq. (4.11), the Wigner function $W(\alpha)$ is real-valued,

$$
W(\alpha)=W(\alpha)^{*} .
$$

We mentioned in I that both the displacement operatos. $\mathrm{D}(\alpha)$ and the operators $T(\alpha, s)$ for Res $\leq 0$ have the property ${ }^{2}$ that if $\tau$ is any trace class operator then the function $\operatorname{Tr}\left[T^{\prime} D(\alpha)\right]$ and $\operatorname{Tr}[T T(\alpha, s)]$ are uniformly continuous functions of $\alpha$. Thus, since every density operator is in the trace class, both the characteristic function $\chi(\xi)$ and the Wigner function $W(\alpha)$ are uniformly continuous functions of $\alpha$. By using Eq. (4.5) and (I.3.6), we find

$$
\int|W(\alpha)|^{2} \pi^{-1} d^{2} \alpha=\operatorname{Tr} \rho^{2} \leq 1,
$$

which shows that the Wigner distribution is a squareintegrable function of $\alpha$ for all density operators $\rho$.

The Hermitian operators $T(\alpha, 0)$ possess the same type of completeness as do the unitary operators $D(\alpha)$. For, according to Eqs. (2.15) and (2.16), we may expand any bounded operator $F$ in the form

$$
F=\int f(\alpha, 0) T(\alpha, 0) \pi^{-1} d^{2} \alpha,
$$

where the weight function $f(\alpha, 0)$, which is given by the trace

$$
f(\alpha, 0)=\operatorname{Tr}[F T(\alpha, 0)],
$$

is unique and square-integrable. We see from Eq. (I.7.25) that this function satisfies the relation

$$
\int|f(\alpha, 0)|^{2} \pi^{-1} d^{2} \alpha=\operatorname{Tr}\left(F^{\dagger} F\right) .
$$

This relation is identical in content to the relation (I.3.14), which characterizes the completeness property of the displacement operators. The expansion (4.10) for the density operator and the relations (4.12) and (4.14) are a special case of Eqs. (4.15)-(4.17).

Let us now note that by using either of the expansions (4.10) and (4.15) we may write the expectation value of any bounded operator $F$ in the form

$$
\operatorname{Tr}(\rho \mathrm{F})=\int W(\alpha) f(\alpha, 0) \pi^{-1} d^{2} \alpha .
$$

We may conclude from the Schwarz inequality for functions (I.3.9) that this integral converges for all bounded operators $F$ and all density operators $\rho$ since, for such operators, the functions $f(\alpha, 0)$ and $W(\alpha)$ are both square-integrable. It will become evident in Sec.
VI that this property of the Wigner distribution is a consequence of the fact that the real part of the order parameter is zero.

In Sec. VII of I we noted that the correspondence between operators and functions $C(0)$ based upon symmetric ordering $s=0$ is the same as the Weyl correspondence, ${ }^{30}$ Eqs. (I.7.30) and (I.7.31). By comparing Eqs. (4.12) and (4.16) with Eq. (2.18), we see that it is this correspondence which associates the functions $W(\alpha)$ and $f(\alpha, 0)$ with the operators $\rho$ and $F$. Thus if the operators $\rho$ and $F$ possess the symmetrically ordered power-series expansions

$$
\rho=\sum_{n, m=0}^{\infty} \rho_{n, m}(0)\left\{\left(a^{\dagger}\right)^{n} a^{m}\right\}_{0}
$$

and

$$
F=\sum_{n, m=0}^{\infty} f_{n, m}(0)\left\{\left(a^{\dagger}\right)^{n} a^{m}\right\}_{0},
$$

then the functions $W(\alpha)$ and $f(\alpha, 0)$ are given by

$$
W(\alpha)=\sum_{n, m=0}^{\infty} \rho_{n, m}(0)\left(\alpha^{*}\right)^{n} \alpha^{m}
$$

and

$$
f(\alpha, 0)=\sum_{n, m=0}^{\infty} f_{n, m}(0)\left(\alpha^{*}\right)^{n} \alpha^{m} .
$$

The functions $W(\alpha)$ and $f(\alpha, 0)$ may be thought of as generating functions for the coefficients of the symmetrically ordered power-series expansions of the operators $\rho$ and $F$. It is a unique property of the Wigner distribution, among all the distribution functions considered in this paper, that in the integral (4.18) for the ensemble average $\operatorname{Tr}(\rho F)$ both the function $W(\alpha)$ representing the density operator and the function $f(\alpha, 0)$ representing the operator $F$ are associated with their respective operators $\rho$ and $F$ by the same correspondence.

The integral expression (4.18) for the expectation value $\operatorname{Tr}(\rho F)$ becomes particularly simple when the operator $F$ is written in symmetric order. The expectation value of the symmetrically ordered product $\left\{\left(a^{\dagger}\right)^{n} a^{m}\right\}_{0}$, for example, is given by the integral

$$
\operatorname{Tr}\left[\rho\left\{\left(a^{\dagger}\right)^{n} a^{m}\right\}_{0}\right]=\int W(\alpha)\left(\alpha^{*}\right)^{n} \alpha^{m} \pi^{-1} d^{2} \alpha .
$$

Three simple illustrations of this relation are

$$
\begin{array}{r}
\frac{1}{2}\left\langle a^{\dagger} a+a a^{\dagger}\right\rangle=\int|\alpha|^{2} W(\alpha) \pi^{-1} d^{2} \alpha, \\
\frac{1}{3}\left\langle a^{\dagger} a^{2}+a a^{\dagger} a+a^{2} a^{\dagger}\right\rangle=\int \alpha^{*} \alpha^{2} W(\alpha) \pi^{-1} d^{2} \alpha,
\end{array}
$$

\footnotetext{
${ }^{30} \mathrm{H}$. Weyl, The Theory of Groups and Quantum Mechanics (Dover Publications, Inc., New York, 1950), pp. 272-276.
} 
and

$$
\operatorname{Tr}(\rho)=1=\int W(\alpha) \pi^{-1} d^{2} \alpha,
$$

which shows that the Wigner function is normalized.

By using Eq. (I.5.14) we may express the ensemble averages of the normally and antinormally ordered products in terms of the Wigner distribution as the integrals

$$
\operatorname{Tr}\left[\rho\left(a^{\dagger}\right)^{n} a^{m}\right]=n !\left(-\frac{1}{2}\right)^{n} \int \alpha^{m-n} L_{n}^{(m-n)}\left(2|\alpha|^{2}\right)
$$$$
\text { and } \quad \times W(\alpha) \pi^{-1} d^{2} \alpha
$$

$$
\begin{array}{r}
\operatorname{Tr}\left[\rho a^{m}\left(a^{\dagger}\right)^{n}\right]=n !\left(\frac{1}{2}\right)^{n} \int \alpha^{m-n} L_{n}{ }^{(m-n)}\left(-2|\alpha|^{2}\right) \\
\times W(\alpha) \pi^{-1} d^{2} \alpha .
\end{array}
$$

A number of properties of the Wigner distribution follow directly from the explicit form of the operator $T(\alpha, 0)$ given by Eq. (4.11). The eigenvalues $e_{n}(0)$ of this Hermitian operator are $2(-1)^{n}$. It follows then from Eq. (4.12) that the Wigner function, being the expectation value of $T(\alpha, 0)$, is bounded according to the inequalities

$$
-2 \leq W(\alpha) \leq 2
$$

for all density operators.

The operator $(-1)^{a \dagger a}=\exp \left(i \pi a^{\dagger} a\right)$ is a reflection operator in the sense indicated by the relations

and

$$
\exp \left(i \pi a^{\dagger} a\right) a \exp \left(-i \pi a^{\dagger} a\right)=-a
$$

$$
\exp \left(i \pi a^{\dagger} a\right) a^{\dagger} \exp \left(-i \pi a^{\dagger} a\right)=-a^{\dagger},
$$

which imply that it also reflects the Hermitian operators $q$ and $p$. By applying these relations to the displacement operator $D(\alpha)$ we find

$$
\exp \left(i \pi a^{\dagger} a\right) D(\alpha) \exp \left(-i \pi a^{\dagger} a\right)=D^{-1}(\alpha),
$$

so that we have

$$
\begin{aligned}
T(\alpha, 0) & =2 D(2 \alpha) \exp \left(i \pi a^{\dagger} a\right) \\
& =2 \exp \left(i \pi a^{\dagger} a\right) D^{-1}(2 \alpha) .
\end{aligned}
$$

By multiplying together these two forms for $T(\alpha, 0)$, we find

$$
[T(\alpha, 0)]^{2}=4 .
$$

By using Eq. (4.33) we may write the Wigner function as

$$
W(\alpha)=2 \operatorname{Tr}\left[\rho D(2 \alpha) \exp \left(i \pi a^{\dagger} a\right)\right],
$$

which, for a state of precisely $n$ quanta, i.e., $\rho=|n\rangle\langle n|$, is simply

$$
W_{n}(\alpha)=2(-1)^{n}\langle n|D(2 \alpha)| n\rangle .
$$

By referring to the explicit form (I.3.30) for this matrix element, we find

$$
W_{n}(\alpha)=2(-1)^{n} L_{n}\left(4|\alpha|^{2}\right) \exp \left(-2|\alpha|^{2}\right),
$$

where $L_{n}(x)$ is a Laguerre polynomial. ${ }^{29}$ Since half of the eigenvalues of the operator $T(\alpha, 0)$ are negative, the Wigner function often assumes negative values as is illustrated by

$$
W_{n}(0)=2(-1)^{n} \text {. }
$$

Among the functions $P(\alpha), W(\alpha)$, and $\langle\alpha|\rho| \alpha\rangle$, only the last, which we discuss in Sec. V, is positive definite for all density operators.

By using Eqs. (4.36) and (4.11), we may write the Wigner distribution in the following additional forms:

$$
\begin{aligned}
W(\alpha) & =2 \sum_{n=0}^{\infty}(-1)^{n}\langle n|\rho D(2 \alpha)| n\rangle \\
& =2 \operatorname{Tr}\left[D^{-1}(\alpha) \rho D(\alpha) \exp \left(i \pi a^{\dagger} a\right)\right] \\
& =2 \sum_{n=0}^{\infty}(-1)^{n}\left\langle n\left|D^{-1}(\alpha) \rho D(\alpha)\right| n\right\rangle .
\end{aligned}
$$

The last two forms make clear that the dependence of the Wigner function $W(\alpha)$ upon the variable $\alpha$ is related to a unitary transformation of the density operator by the displacement operator $D(\alpha)$. By applying Eq. (I.6.18) to Eq. (3.13), we may similarly interpret the $\alpha$ dependence of the weight function $P(\alpha)$ of the $P$ representation.

\section{THE FUNCTION $\langle\boldsymbol{\alpha}|\boldsymbol{\varrho}| \boldsymbol{\alpha}\rangle$}

In Secs. III and IV we considered distribution functions that are useful for finding the expectation values of operators written in normal or symmetric order. We turn now to a function that may be used to express the ensemble averages of antinormally ordered operators as simple integrals.

Let us suppose that the operator $F$ possesses the antinormally ordered power-series expansion

$$
F=\sum_{n, m=0}^{\infty} f_{n, m}(-1) a^{m}\left(a^{\dagger}\right)^{n} .
$$

Then by proceeding as in Eqs. (3.9)-(3.11), we may write the operator $F$ in the form

$$
F=\int f(\alpha, 1)|\alpha\rangle\langle\alpha| \pi^{-1} d^{2} \alpha,
$$

where the function $f(\alpha, 1)$ is given by

$$
f(\alpha, 1)=\sum_{n, m=0}^{\infty} f_{n, m}(-1)\left(\alpha^{*}\right)^{n} \alpha^{m} .
$$

By referring to Eq. (2.18), we see that this function may be expressed in terms of the operator $T(\alpha, 1)$ as the trace

$$
f(\alpha, 1)=\operatorname{Tr}[F T(\alpha, 1)] .
$$

The appearance within this trace of the operator $T(\alpha, 1)$ all of whose eigenvalues are infinite reflects the fact 
that antinormally ordered power series do not exist for all operators $F$.

We may use the expansion (5.2) to write the expectation value of the operator $F$ in the form

$$
\begin{aligned}
\operatorname{Tr}(\rho F) & =\int f(\alpha, 1) \operatorname{Tr}[\rho|\alpha\rangle\langle\alpha|] \pi^{-1} d^{2} \alpha \\
& =\int f(\alpha, 1)\langle\alpha|\rho| \alpha\rangle \pi^{-1} d^{2} \alpha .
\end{aligned}
$$

Thus the function $\langle\alpha|\rho| \alpha\rangle$ has for antinormally ordered operators the useful property ${ }^{8,9,18}$ exhibited in Eqs. (3.7) and (4.18) by the functions $P(\alpha)$ and $W(\alpha)$ for the cases of normally and symmetrically ordered operators.

The function $\langle\alpha|\rho| \alpha\rangle$ clearly is non-negative and bounded according to the relation

Its normalization,

$$
0 \leq\langle\alpha|\rho| \alpha\rangle \leq 1 .
$$

$$
1=\operatorname{Tr} \rho=\int\langle\alpha|\rho| \alpha\rangle \pi^{-1} d^{2} \alpha,
$$

is evident from Eq. (I.2.28). By recalling that the operator $T(\alpha,-1)$ is a projection operator upon the coherent state $|\alpha\rangle$, as shown by Eq. (3.16), we may write the function $\langle\alpha|\rho| \alpha\rangle$ in the form

$$
\langle\alpha|\rho| \alpha\rangle=\operatorname{Tr}[\rho T(\alpha,-1)] .
$$

This relation is the analog for antinormal order, $s=-1$, of Eqs. (3.13) and (4.12) for the functions $P(\alpha)$ and $W(\alpha)$.

By using Eqs. (5.4) and (5.9) we may write Eq. (5.6) in the form

$$
\operatorname{Tr}(\rho F)=\int \operatorname{Tr}[F T(\alpha, 1)] \operatorname{Tr}[\rho T(\alpha,-1)] \pi^{-1} d^{2} \alpha .
$$

If we now contrast this relation with its counterpart (3.18) for the case of the $P$ representation, we see that the roles of the operators $T(\alpha, 1)$ and $T(\alpha,-1)$ have been interchanged. In the corresponding relation for the case of the Wigner distribution, Eq. (4.18), both $T(\alpha,-1)$ and the singular operator $T(\alpha, 1)$ are replaced by $T(\alpha, 0)$ whose eigenvalues are \pm 2 . For this reason only the Wigner function of the three quasiprobability distributions considered thus far may be used to write the ensemble averages of all bounded operators as convergent integrals.

We now note the very limited sense in which the function $\langle\alpha|\rho| \alpha\rangle$ is a weight function for the density operator. By using Eqs. (5.9), (2.15) and (2.16) we may formally express the density operator as the integral

$$
\rho=\int\langle\alpha|\rho| \alpha\rangle T(\alpha, 1) \pi^{-1} d^{2} \alpha .
$$

The appearance in this expansion of the singular operator $T(\alpha, 1)$ may be interpreted in terms of the properties of the function $\langle\alpha|\rho| \alpha\rangle$. As we have noted, this function is bounded, non-negative, and normalized. It is also an infinitely differentiable function, as may be seen by using Eq. (I.2.23) to expand the coherent states $|\alpha\rangle$ and $\langle\alpha|$ in powers of $\alpha$ and $\alpha^{*}$. The function $\langle\alpha|\rho| \alpha\rangle$ thus has all the properties that one would expect of the smoothest classical probability distribution. The expansion (5.11) therefore suggests the interpretation that only so singular an operator as $T(\alpha, 1)$ can be used to construct arbitrary density operators when weighted with so well behaved a weight function as $\langle\alpha|\rho| \alpha\rangle$. The opposite interpretation evidently applies to the $P$ representation, in which the roles of the operators $T(\alpha, 1)$ and $T(\alpha,-1)=|\alpha\rangle\langle\alpha|$ are interchanged. It appears, then, that the nonexistence of the $P$ representation for certain density operators is a consequence of the extreme "smoothness" of the coherent state projection operator $|\alpha\rangle\langle\alpha|$.

In analogy with Eqs. (3.20) and (4.4), let us define the antinormally ordered characteristic function $\chi_{A}(\xi)$ as the expectation value

$$
\begin{aligned}
\chi_{A}(\xi) & =\operatorname{Tr}[\rho D(\xi,-1)] \\
& =\operatorname{Tr}\left[\rho \exp \left(-\xi^{*} a\right) \exp \left(\xi a^{\dagger}\right)\right] .
\end{aligned}
$$

By using Eqs. (2.7)-(2.9) we see that the characteristic functions $\chi_{N}(\xi), \chi(\xi)$, and $\chi_{A}(\xi)$ stand in the simple relationship

$$
\chi_{N}(\xi)=e^{|\xi|^{2} / 2} \chi(\xi)=e^{|\xi|^{2}} \chi_{A}(\xi) .
$$

Since, as we noted in Sec. IV, the function $\chi(\xi)$ is a continuous function of $\xi$, it follows that all three characteristic functions are continuous. We see from Eq. (4.6) that the modulus of the antinormally ordered characteristic function $\chi_{A}(\xi)$ is bounded by

$$
\left|\chi_{A}(\xi)\right| \leq e^{-|\xi|^{2 / 2}} \text {. }
$$

If we use Eq. (5.6) to evaluate the antinormally ordered expectation value (5.12), then we find

$$
\chi_{A}(\xi)=\int \exp \left(\xi \alpha^{*}-\xi^{*} \alpha\right)\langle\alpha|\rho| \alpha\rangle \pi^{-1} d^{2} \alpha,
$$

which shows that the function $\chi_{A}(\xi)$ is the complex Fourier transform of the function $\langle\alpha|\rho| \alpha\rangle$. By means of Eq. (I.3.4) and (I.3.5) we obtain the inverse relation

$$
\langle\alpha|\rho| \alpha\rangle=\int \exp \left(\alpha \xi^{*}-\alpha^{*} \xi\right) \chi_{A}(\xi) \pi^{-1} d^{2} \xi .
$$

As a simple illustration of Eq. (5.6), we note that the expectation values of the antinormally ordered products can be written in the form

$$
\operatorname{Tr}\left[\rho a^{m}\left(a^{\dagger}\right)^{n}\right]=\int\left(\alpha^{*}\right)^{n} \alpha^{m}\langle\alpha|\rho| \alpha\rangle \pi^{-1} d^{2} \alpha .
$$


The function $\langle\alpha|\rho| \alpha\rangle$. may also be used to evaluate the mean values of normally and symmetrically ordered products. By using Eq. (I.5.14) we may write them as the integrals

$$
\begin{array}{r}
\operatorname{Te}\left[\rho\left(a^{\dagger}\right)^{n} a^{m}\right]=(-1)^{n} n ! \int a^{m-n} L_{n}{ }^{(m-n)}\left(|\alpha|^{2}\right) \\
\times\langle\alpha|\rho| \alpha\rangle \pi^{-1} d^{2} \alpha
\end{array}
$$

and

$$
\begin{array}{r}
\operatorname{Tr}\left[\rho\left\{\left(a^{\dagger}\right)^{n} a^{m}\right\}_{0}\right]=\left(-\frac{1}{2}\right)^{n} n ! \int \alpha^{m-n} L_{n}{ }^{(m-n)}\left(2|\alpha|^{2}\right) \\
\times\langle\alpha|\rho| \alpha\rangle \pi^{-1} d^{2} \alpha .
\end{array}
$$

In Sec. IV of I we showed that normally ordered power series exist and converge for virtually all operators of interest, i.e., for those that satisfy the condition (I.4.23). In particular, every density operator $\rho$ possesses the expansion

$$
\rho=\sum_{n, m=0}^{\infty} \rho_{n, m}(1)\left(a^{\dagger}\right)^{n} a^{m},
$$

and by using the eigenvalue property of the coherent states we find

$$
\langle\alpha|\rho| \alpha\rangle=\sum_{n, m=0}^{\infty} \rho_{n, m}(1)\left(\alpha^{*}\right)^{n} a^{m} .
$$

Thus the function $\langle\alpha|\rho| \alpha\rangle$ is the one associated with the density operator $\rho$ by the correspondence $C(1)$ based upon normal ordering. Equivalently, the function $\langle\alpha|\rho| \alpha\rangle$ is a generating function for the coefficients of the normally ordered power-series expansion of the density operator. The convergence of the series (5.20) and (5.21) is consistent with the fact, mentioned earlier, that the function $\langle\alpha|\rho| \alpha\rangle$ is infinitely differentiable. We note that Eqs. (5.20) and (5.21) correspond to Eqs. (3.10) and (3.11) for the weight function $P(\alpha)$ and to Eqs. (4.19) and (4.21) for the Wigner distribution $W(\alpha)$.

Let us now define the function $R\left(\alpha^{*}, \beta\right)$ by the relation $^{6}$

$$
R\left(\alpha^{*}, \beta\right)=e^{\left(|\alpha|^{2}+|\beta|^{2}\right) / 2}\langle\alpha|\rho| \beta\rangle .
$$

The function $R\left(\alpha^{*}, \beta\right)$ is an entire function of the two complex variables $\alpha^{*}$ and $\beta$. By twice using Eq. (I.2.27), we may write the density operator in the form

$$
\rho=\int|\alpha\rangle\langle\beta|\langle\alpha|\rho| \beta\rangle \pi^{-2} d^{2} \alpha d^{2} \beta .
$$

The function $R\left(\alpha^{*} \beta\right)$ therefore affords for every density operator $\rho$ the two-variable representation

$$
\rho=\int|\alpha\rangle\langle\beta| R\left(\alpha^{*}, \beta\right) e^{-\left(|\alpha|^{2}+|\beta|^{2}\right) / 2} \pi^{-2} d^{2} \alpha d^{2} \beta,
$$

which has been called the $R$ representation. The relationship between the functions $R\left(\alpha^{*}, B\right)$ and $\langle\alpha|\rho| \alpha\rangle$ is given by

$$
\langle\alpha|\rho| \alpha\rangle=e^{-|\alpha|^{2}} R\left(\alpha^{*}, \alpha\right) .
$$

\section{ENSEMBLE AVERAGES AND $S$ ORDERING}

In the present section we draw together the results of the Secs. III-V by making use of the parametrized ordering convention introduced in I. We define a general representation for the density operator in which the weight function $W(\alpha, s)$ may be identified with the weight functions $\langle\alpha|\rho| \alpha\rangle, W(\alpha)$, and $P(\alpha)$ when the order parameter $s$ assumes the values $s=-1,0$, and +1 , respectively. In characterizing the behavior of the function $W(\alpha, s)$ we will see the unfolding of an orderly progression of mathematical properties as the parameter $s$ is advanced from $s=-1$ corresponding to the function $\langle\alpha|\rho| \alpha\rangle$ to $s=+1$ corresponding to the function $P(\alpha)$.

In I we introduced for every bounded operator $F$ the representation

$$
F=\int f(\alpha,-s) T(\alpha, s) \pi^{-1} d^{2} \alpha,
$$

in which the weight function $f(\alpha,-s)$ is given by

$$
f(\alpha,-s)=\operatorname{Tr}[F T(\alpha,-s)] .
$$

The operator $F$ and its weight function $f(\alpha,-s)$ are associated by the correspondence $C(s)$ which is based upon the power-series expansions

$$
F=\sum_{n, m=0}^{\infty} f_{n, m}(s)\left\{\left(a^{\dagger}\right)^{n} a^{m}\right\}_{s}
$$

and

$$
f(\alpha,-s)=\sum_{n, m=0}^{\infty} f_{n, m}(s)\left(\alpha^{*}\right)^{n} \alpha^{m} .
$$

By multiplying both sides of the expansion (6.1) by an arbitrary density operator $\rho$ and forming the trace of the resulting relation we find

$$
\operatorname{Tr}(\rho F)=\int f(\alpha,-s) \operatorname{Tr}[\rho T(\alpha, s)] \pi^{-1} d^{2} \alpha .
$$

Let us introduce the function $W(\alpha, s)$ as the expectation value of the operator $T(\alpha, s)$,

$$
W(\alpha, s)=\operatorname{Tr}[\rho T(\alpha, s)] .
$$

This function permits us to express the expectation value of the operator $F$ as the integral

$$
\operatorname{Tr}(\rho F)=\int f(\alpha,-s) W(\alpha, s) \pi^{-1} d^{2} \alpha,
$$

in which $F$ is represented by the function $f(\alpha,-s)$ associated with it by the correspondence $C(s)$. 
By referring to Eqs. (6.1) and (6.2) we find that the function $W(\alpha, s)$ is also the weight function for the density operator $\rho$ in the expansion

$$
\rho=\int W(\alpha, s) T(\alpha,-s) \pi^{-1} d^{2} \alpha .
$$

This relation, we note, affords an alternative way of deriving the integral expression (6.7) for the ensemble average $\operatorname{Tr}[\rho F]$.

By comparing the definition (6.6) with Eqs. (3.13), (4.12), and (5.9) we may identify the function $W(\alpha, s)$ for $s=-1,0$, and +1 with the functions $\langle\alpha|\rho| \alpha\rangle$, $W(\alpha)$, and $\pi P(\alpha)$, respectively :

$$
\begin{aligned}
\langle\alpha|\rho| \alpha\rangle & =W(\alpha,-1), \\
W(\alpha) & =W(\alpha, 0), \\
P(\alpha) & =\pi^{-1} W(\alpha, 1) .
\end{aligned}
$$

If in Eqs. (6.7) and (6.8) we let $s=-1,0,+1$, we then obtain, respectively, Eqs. (5.6) and (5.11) for the case of the function $\langle\alpha|\rho| \alpha\rangle$, Eqs. (4.18) and (4.10) for the case of the Wigner distribution $W(\alpha)$, and Eqs. (3.3) and (3.2) for the case of the $P$ representation.

By comparing the definition (6.6) with Eqs. (6.3) and (6.4), we see that the function $W(\alpha, s)$ and the density operator $\rho$ are related by the correspondence $C(-s)$ :

$$
\begin{aligned}
\rho & =\sum_{n, m=0}^{\infty} \rho_{n, m}(-s)\left\{\left(a^{\dagger}\right)^{n} a^{m}\right\}_{-s}, \\
W(\alpha, s) & =\sum_{n, m=0}^{\infty} \rho_{n, m}(-s)\left(\alpha^{*}\right)^{n} \alpha^{m} .
\end{aligned}
$$

Thus in the integral (6.7) for the ensemble average $\operatorname{Tr}[\rho F]$ the functions $f(\alpha,-s)$ and $W(\alpha, s)$ bear to the operator $F$ and $\rho$ the relationships $C(s)$ and $C(-s)$, respectively. If in Eqs. (6.12) and (6.13) we put $s=-1,0,+1$, then we may recover the earlier relations (5.20), (5.21), (4.19), (4.21), (3.10), and (3.11), which obtain for normal, symmetric, and antinormal ordering.

A simple illustration of the weight function $W(\alpha, s)$ is provided by the density operator for a pure coherent state, $\rho=|\beta\rangle\langle\beta|$. According to the definition (6.6) the function $W(\alpha, s)$ is given by the matrix element

$$
W(\alpha, s)=\langle\beta|T(\alpha, s)| \beta\rangle,
$$

which is evaluated in Eq. (I.6.36). By using that result we find

$$
W(\alpha, s)=\frac{2}{1-s} \exp \left(-\frac{2|\alpha-\beta|^{2}}{1-s}\right),
$$

which shows that the function $W(\alpha, s)$ has an essential singularity at $s=1$ and is for Res $<1$ a Gaussian exponential with a maximum at $\alpha=\beta$. By referring to Eqs. (6.9)-(6.11) we see that the weight functions $\langle\alpha|\rho| \alpha\rangle, W(\alpha)$, and $P(\alpha)$ are given by

$$
\begin{aligned}
\langle\alpha|\rho| \alpha\rangle & =e^{-|\alpha-\beta|^{2}}, \\
W(\alpha) & =2 e^{-2|\alpha-\beta|^{2}}, \\
P(\alpha) & =\delta^{(2)}(\alpha-\beta),
\end{aligned}
$$

where the last expression is obtained by using the limiting process (3.15) for $P(\alpha)$.

By using Eq. (I.6.16) we find

$$
W\left(\alpha, s^{*}\right)=W(\alpha, s)^{*},
$$

which shows that the function $W(\alpha, s)$ is real for real values of $s$. By using either the general expression (6.7) or the relation (I.6.45), we may write the expectation values of the $s$-ordered products as the integrals

$$
\operatorname{Tr}\left[\rho\left\{\left(a^{\dagger}\right)^{n} a^{m}\right\}_{s}\right]=\int\left(\alpha^{*}\right)^{n} \alpha^{m} W(\alpha, s) \pi^{-1} d^{2} \alpha .
$$

This expression is a generalization to arbitrary $s$ of Eqs. (5.17), (4.23), and (1.3) which apply for $s=-1$, $0,+1$, respectively. By setting $n=m=0$ in this relation, we find, as the normalization condition for $W(\alpha, s)$,

$$
1=\operatorname{Tr} \rho=\int W(\alpha, s) \pi^{-1} d^{2} \alpha .
$$

The expectation value of the $s$-ordered displacement operator $D(\xi, s)$ may be obtained from Eqs. (I.7.11) and (6.7) as the integral

$$
\operatorname{Tr}[\rho D(\xi, s)]=\int e^{\xi \alpha^{*}-\xi^{*} \alpha} W(\alpha, s) \pi^{-1} d^{2} \alpha .
$$

Let us define what we may call the $s$-ordered characteristic function $\chi(\xi, s)$ by the relation

$$
\chi(\xi, s)=\operatorname{Tr}[\rho D(\xi, s)]=e^{s|\xi|^{2} / 2 \chi}(\xi),
$$

where $\chi(\xi)$ is the characteristic function introduced in Sec. IV. We see from Eq. (6.21) that it is the complex Fourier transform of the function $W(\alpha, s)$,

$$
\chi(\xi, s)=\int e^{\xi \alpha^{*}-\xi^{*} \alpha} W(\alpha, s) \pi^{-1} d^{2} \alpha,
$$

and by referring to Eqs. (I.3.4) and (I.3.5) we find as the inverse relation

$$
W(\alpha, s)=\int e^{\alpha \xi^{*}-\alpha^{*} \xi} \chi(\xi, s) \pi^{-1} d^{2} \xi .
$$

These Fourier transform relations generalize to arbitrary $s$ the earlier relations (5.15) and (5.16), (4.8) and (4.9), and (3.21) and (3.22), which are realized when $s=-1,0,+1$.

We noted in Sec. IV that the function $\chi(\xi)$ is a uniformly continuous function that is bounded by 
unity. The function $\chi(\xi, s)$ is therefore a continuous function of $\xi$ and, of course, an entire function of $s$. It is bounded by

$$
|\chi(\xi, s)| \leq \exp \left(\frac{1}{2} \operatorname{Res}|\xi|^{2}\right) .
$$

By using Eqs. (6.19) and (6.22), we easily find the relation

$$
\operatorname{Tr}\left[\rho\left\{\left(a^{\dagger}\right)^{n} a^{m}\right\}_{s}\right]=\left.\frac{\partial^{n+m} \chi(\xi, s)}{\partial \xi^{n} \partial\left(-\xi^{*}\right)^{m}}\right|_{\xi=0} .
$$

According to Eq. (I.6.28) the eigenvalues $e_{n}(s)$ of the operator $T(\alpha, s)$ are given by

$$
e_{n}(s)=\frac{2}{1-s}\left(\frac{s+1}{s-1}\right)^{n}
$$

We note that they are all positive and less than unity for $s$ real and $<-1$, with the limiting values $e_{n}(-1)$ $=\delta_{n, 0}$. Thus the function $W(\alpha, s)$, being the expectation value of $T(\alpha, s)$, satisfies the inequality

$$
0 \leq W(\alpha, s) \leq 2 /(1-s)
$$

for $s$ real and $\leq-1$. By examining $e_{0}(s)$ and $e_{1}(s)$ we see, furthermore, that the full set of eigenvalues is not positive unless $s$ is real and $\leq-1$. The function $W(\alpha, s)$ is therefore positive definite for all density operators only when $s$ is real and $\leq-1$.

Since every density operator is in the trace class, we know from the analysis of Sec. VII of I that the function $W(\alpha, s)$ is a uniformly continuous function of $\alpha$ for Res $\leq 0$. By using Eq. (I.7.43) and the fact that $\operatorname{Tr} \rho=1$, we find that the modulus of the function $W(\alpha, s)$ is bounded by

$$
|W(\alpha, s)| \leq 2 /|1-s|
$$

for $\operatorname{Re} s \leq 0$.

Since every density operator is a bounded operator, we may obtain from Eq. (I.7.38) the relation

$$
1 \geq \operatorname{Tr} \rho^{2} \geq \int|W(\alpha, s)|{ }^{2} \pi^{-1} d^{2} \alpha
$$

for $\operatorname{Re} \leq \leq 0$, in which the first and second inequalities are equalities only when the density operator represents a pure state and when $\operatorname{Re} s=0$, respectively. From the analysis of Sec. VII of I we may assert further that the function $W(\alpha, s)$ is analytic in $s$ and infinitely differentiable with respect to $\alpha$ and $\alpha^{*}$ throughout the half-plane $\operatorname{Re} s<0$. The function $W(\alpha, s)$ may be represented by a power series ${ }^{2}$ in the variables $s, \alpha$, and $\alpha^{*}$ for $\operatorname{Res}<0$.

As the line $\operatorname{Re}=0$ is crossed, however, the function $W(\alpha, s)$ retains, in general, none of the properties which we have observed in the half-plane $\operatorname{Re} s \leq 0$. As we noted in Sec. VII of I, for each density operator $\rho$ there is a non-negative number $x(\rho)$ such that the function $W(\alpha, s)$ remains square-integrable and infinitely dif- ferentiable for $\operatorname{Re} s<x(\rho)$, but not for larger values of Res. Our examples in Sec. VII will show that the quantity $x(\rho)$ is arbitrarily small for certain classes of denisty operators. The function $W(\alpha, s)$ typically has a singularity of some sort on the line Res $=x(\rho)$ and beyond that singularity it lies outside the class of tempered distributions. $^{2}$ In Sec. III we mentioned the result that the weight function $P(\alpha)$ of the $P$ representation is singular for all pure states. Thus for such density operators the quantity $x(\rho)$ lies in the interval

$$
0 \leq x(\rho) \leq 1
$$

The dependence of the function $W(\alpha, s)$ upon the parameter $s$ is expressed by the convolution law (I.7.32), according to which we have

$$
W(\alpha, s)=\frac{2}{t-s} \int \exp \left(-\frac{2|\alpha-\beta|^{2}}{t-s}\right) W(\beta, t) \frac{d^{2} \beta}{\pi}
$$

for Res $<$ Ret. This Gaussian convolution tends to smooth out any unruly behavior of the function $W(\beta, t)$. It shows clearly why the quasiprobability distribution $W(\alpha, s)$ becomes progressively better behaved as Res decreases. We note also from Eq. (6.32) that the function $W(\alpha, s)$ obeys a second-order partial differential equation analogous to the heat-diffusion equation. This property leads to an instructive analogy which we take up in Sec. IX.

The convolution law (6.32) provides us with a number of integral relations between the functions considered in Secs. III-V. If we assume that the $P$ representation exists for the density operator $\rho$, then by letting $t=+1$, and $s=0$ and -1 we may express the Wigner function $W(\alpha)$ and the function $\langle\alpha|\rho| \alpha\rangle$ as the integrals ${ }^{8}$

$$
W(\alpha)=2 \int e^{-2|\alpha-\beta|^{2}} P(\beta) d^{2} \beta
$$

and

$$
\langle\alpha|\rho| \alpha\rangle=\int e^{-|\alpha-\beta|^{2}} P(\beta) d^{2} \beta .
$$

Similarly, since the Wigner function $W(\alpha)$ exists for all density operators $\rho$, we have in general the relation ${ }^{2}$

$$
\langle\alpha|\rho| \alpha\rangle=2 \int e^{-\left.2|\alpha-\beta|\right|^{2}} W(\alpha) \pi^{-1} d^{2} \beta .
$$

Let us now suppose that we wish to find the ensemble average of the $s$-ordered product $\left\{\left(a^{\dagger}\right)^{n} a^{m}\right\}_{s}$ but that we know only the function $W(\alpha, t)$ and not the function $W(\alpha, s)$ or, alternatively, that the function $W(\alpha, s)$ is too singular for the relation (6.19) to apply. We may in such a case use the relations (I.5.13) and (I.5.14) to express the $s$-ordered product in terms of $t$-ordered products and so obtain the desired expectation value 
as the integrals

$$
\begin{aligned}
\operatorname{Tr}\left[\rho\left\{\left(a^{\dagger}\right)^{n} a^{m}\right\}_{s}\right] & =m !\left(\frac{t-s}{2}\right)^{m} \int\left(\alpha^{*}\right)^{n-m} \\
& \times L_{m}{ }^{(n-m)}\left(\frac{2|\alpha|^{2}}{s-t}\right) W(\alpha, t) \pi^{-1} d^{2} \alpha \\
& =n !\left(\frac{t-s}{2}\right)^{n} \int \alpha^{m-n} \\
& \times L_{n}{ }^{(m-n)}\left(\frac{2|\alpha|^{2}}{s-t}\right) W(\alpha, t) \pi^{-1} d^{2} \alpha .
\end{aligned}
$$

\section{EXAMPLES OF THE GENERAL QUASI- PROBABILITY FUNCTION $W(\alpha, s)$}

We now illustrate the results of the preceding sections by constructing the function $W(\alpha, s)$ for some simple density operators and by using it to evaluate the ensemble averages of a variety of observables. We have shown in Sec. VI that the function $W(\alpha, s)$ is a wellbehaved function of both $\alpha$ and $s$ when the real part of $s$ is negative. The examples of the present section will verify this behavior and will illustrate some of the singularities that occur for $\operatorname{Re} s>0$.

By referring to Eqs. (I.6.17), (6.27), and (I.6.38), we see that for $t$ real and $\leq-1$ each operator $T(\gamma, t)$ is Hermitian, positive definite, and of unit trace. The operators $T(\gamma, t)$, which we have introduced for other purposes, therefore form for $t \leq-1$ a two-parameter family of density operators.

This family of density operators in fact describes a broad class of physically important fields. For $t=-1$ the operator $T(\gamma,-1)$ describes a coherent field of amplitude $\gamma$,

$$
T(\gamma,-1)=|\gamma\rangle\langle\gamma|
$$

as is shown by Eq. (I.6.29). According to Eq. (I.6.26) it characterizes at the other extreme, when $\gamma=0$ and $t \leq-1$, the general chaotic field ${ }^{6}$

$$
T(0, t)=\frac{1}{\langle n\rangle+1} \sum_{n=0}\left(\frac{\langle n\rangle}{\langle n\rangle+1}\right)^{n}|n\rangle\langle n|,
$$

in which the mean number of quanta $\langle n\rangle$ is given by

$$
\langle n\rangle=-\frac{1}{2}(t+1) \text {. }
$$

The operator $T(\gamma, t)$, regarded as a density operator, describes more generally the field that results from the superposition of a coherent signal of amplitude $\gamma$,

$$
T(\gamma, t)=D(\gamma) T(0, t) D^{-1}(\gamma),
$$

upon a chaotic field with a mean member of quanta $\langle n\rangle=-\frac{1}{2}(t+1)$. By using Eq. (I.6.25) we may write the density operator $T(\gamma, t)$ in the suggestive form

$$
T(\gamma, t)=\left(1-e^{-\beta}\right) \exp [-\beta \Re(\gamma)],
$$

where

$$
\beta(t)=-\ln \left(\frac{t+1}{t-1}\right)
$$

and

$$
\begin{aligned}
\Re(\gamma) & =D(\gamma) a^{\dagger} a D^{-1}(\gamma) \\
& =a^{\dagger} a-\gamma a^{\dagger}-\gamma^{*} a+|\gamma|^{2} .
\end{aligned}
$$

For $t \leq-1$ the parameter $\beta$ is real and positive with the limiting value of $\beta=\infty$ for $t=-1$. The operator (7.5) for $\gamma=0$ is of the type which describes the thermal equilibrium state of an electromagnetic field mode. If this state is exposed to the radiation from a prescribed, $c$-number current distribution then the appropriate density operator ${ }^{31}$ is given by Eq. (7.5), with $\gamma \neq 0$.

By using Eq. (I.6.39) we may obtain the function $W(\alpha, s)$ for the density operator $T(\gamma, t)$ as the trace

$$
\begin{aligned}
W(\alpha, s) & =\operatorname{Tr}[T(\gamma, t) T(\alpha, s)] \\
& =-\frac{2}{t+s} \exp \left(\frac{2|\alpha-\gamma|^{2}}{t+s}\right) \\
& =\frac{2}{2\langle n\rangle+1-s} \exp \left(\frac{-2|\alpha-\gamma|^{2}}{2\langle n\rangle+1-s}\right) .
\end{aligned}
$$

The weight function $P(\alpha)$ of the $P$ representation, the Wigner distribution $W(\alpha)$, and the function $W(\alpha,-1)$ correspond to the values $s=1,0,-1$ and are given by

$$
\begin{gathered}
W(\alpha, 1)=\pi P(\alpha)=\frac{1}{\langle n\rangle} \exp \left(-\frac{|\alpha-\gamma|^{2}}{\langle n\rangle}\right), \\
W(\alpha, 0)=W(\alpha)=\frac{1}{\langle n\rangle+\frac{1}{2}} \exp \left(-\frac{|\alpha-\gamma|^{2}}{\langle n\rangle+\frac{1}{2}}\right), \\
W(\alpha,-1)=\langle\alpha|\rho| \alpha\rangle=\frac{1}{\langle n\rangle+1} \exp \left(-\frac{|\alpha-\gamma|^{2}}{\langle n\rangle+1}\right) .
\end{gathered}
$$

For the density operator $T(\gamma, t)$ the function $W(\alpha, s)$ has an essential singularity at $s=-t=1+2\langle n\rangle$. The parameter $x(\rho)$, introduced in Sec. VI, clearly assumes the value

$$
x(\rho)=-t=2\langle n\rangle+1 \geq 1 .
$$

The function $W(\alpha, s)$ is a tempered distribution on the line $\operatorname{Re} s=x(\rho)$ but not for larger values of $\operatorname{Res}$, where it increases as an exponential function of $|\alpha-\gamma|^{2}$.

The family of density operators $T(\gamma, t)$ provides a physical characterization of the fields which may be described by means of the $P$ representation. For if $\rho$ is a density operator for which the function $W(\alpha, s)$ is

\footnotetext{
${ }^{31}$ R. J. Glauber, Phys. Rev. 84, 395 (1951).
} 
well behaved for Res $\geq 1$, then by using Eqs. (6.8) and (7.5) we may write it in the form

$$
\begin{aligned}
\rho=\left(1-e^{-\beta(-s)}\right) \int W(\alpha, s) \\
\quad \times \exp [-\beta(-s) \Re(\alpha)] \pi^{-1} d^{2} \alpha,
\end{aligned}
$$

where $s \geq 1$. Such a density operator is therefore a linear combination, or, if $W(\alpha, s) \geq 0$, a statistical average of the density operators $T(\alpha,-s)$ which describe the superposition of a coherent excitation upon a thermal equilibrium state. We see from Eq. (7.6) that the larger the value of $s$ for which the function $W(\alpha, s)$ is regular, the higher are the temperature and entropy of the thermal or chaotic component of the mixtures (7.5) which may appear in the expansion (7.13).

For the density operator $T(\gamma, t)$ the ensemble average of any operator $F$ is the function $f(\gamma, t)$ associated with $F$ by the correspondence $C(-t)$,

$$
\operatorname{Tr}[T(\gamma, t) F]=f(\gamma, t),
$$

as shown by Eq. (6.2). Thus in particular by referring to Eq. (I.5.14) we secure for the expectation value of the general s-ordered product $\left\{\left(a^{\dagger}\right)^{n} a^{m}\right\}_{s}$ the result

$$
\begin{aligned}
& \operatorname{Tr}\left[T(\gamma, t)\left\{\left(a^{\dagger}\right)^{n} a^{m}\right\}_{s}\right] \\
& \quad=n !\left(-\frac{t+s}{2}\right)^{n} \gamma^{m-n} L_{n}(m-n) \\
&
\end{aligned}
$$

In terms of the mean number of quanta $\langle n\rangle$ of the chaotic component of the field, this ensemble average may be written as

$$
\begin{aligned}
\operatorname{Tr}\left[T(\gamma, t)\left\{\left(a^{\dagger}\right)^{n} a^{m}\right\}_{s}\right] & \\
= & n !\langle n\rangle^{n}\left(1+\frac{1-s}{2\langle n\rangle}\right)^{n} \gamma^{m-n} \\
& \times L_{n}{ }^{(m-n)}\left[-\frac{|\gamma|^{2}}{\langle n\rangle}\left(1+\frac{1-s}{2\langle n\rangle}\right)^{-1}\right] .
\end{aligned}
$$

When the coherent signal or prescribed current vanishes, i.e., $\gamma$ is zero, we have

$$
\operatorname{Tr}\left[T(0, t)\left\{\left(a^{\dagger}\right)^{n} a^{m}\right\}_{s}\right]=\delta_{n, m} n !\langle n\rangle^{n}\left(1+\frac{1-s}{2\langle n\rangle}\right)^{n} .
$$

For large numbers of quanta, i.e., $\langle n\rangle \gg|1-s|$, we have the approximation

$$
\begin{aligned}
\operatorname{Tr}\left[T(0, t)\left\{\left(a^{\dagger}\right)^{n} a^{m}\right\}_{s}\right] & \\
& \sim \delta_{n, m} n !\langle n\rangle^{n}\left[1+\frac{n}{\langle n\rangle}\left(\frac{1-s}{2}\right)\right] .
\end{aligned}
$$

For the case of normal ordering, $s=1$, Eq. (7.17) becomes

$$
\operatorname{Tr}\left[T(0, t)\left(a^{\dagger}\right)^{n} a^{m}\right]=\delta_{n, m} n !\langle n\rangle^{n} .
$$

We note that the general expectation value (7.16) factors into a part referring to the chaotic field alone and a part representing the effect of the coherent signal of amplitude $\gamma$ :

$$
\begin{aligned}
& \operatorname{Tr}\left[T(\gamma, t)\left\{\left(a^{\dagger}\right)^{n} a^{m}\right\}_{s}\right] \\
& =\operatorname{Tr}\left[T(0, t)\left\{\left(a^{\dagger}\right)^{n} a^{m}\right\}_{s}\right] \gamma^{m-n} \\
& \quad \times L_{n}{ }^{(m-n)}\left[-\frac{|\gamma|^{2}}{\langle n\rangle}\left(1+\frac{1-s}{2\langle n\rangle}\right)^{-1}\right] .
\end{aligned}
$$

By using the definition ${ }^{29}$ of the associated Laguerre polynomial, we find for high signal-to-noise ratios, i.e., for $\left.\left.|\gamma|{ }^{2}\right\rangle\right\rangle\langle n\rangle$, that the two leading terms of the Laguerre polynomial yield

$\operatorname{Tr}\left[T(\gamma, t)\left\{\left(a^{\dagger}\right)^{n} a^{m}\right\}_{s}\right]$

$$
\sim\left(\gamma^{*}\right)^{n} \gamma^{m}\left[1+\frac{n m\langle n\rangle}{|\gamma|^{2}}\left(1+\frac{1-s}{2\langle n\rangle}\right)\right] .
$$

Specializing now, first to the case of normal ordering, $s=1$, and then to the case of a pure coherent field, $t=1$, we find

$$
\operatorname{Tr}\left[T(\gamma, t)\left(a^{\dagger}\right)^{n} a^{m}\right] \sim\left(\gamma^{*}\right)^{n} \gamma^{m}\left(1+n m\langle n\rangle /|\gamma|^{2}\right)
$$

and

$$
\operatorname{Tr}\left[T(\gamma,-1)\left(a^{\dagger}\right)^{n} a^{m}\right]=\left(\gamma^{*}\right)^{n} \gamma^{m},
$$

respectively.

According to Eq. (I.6.51), the function $W(\alpha, s)$ for the pure state of precisely $n$ quanta, $\rho=|n\rangle\langle n|$, is given by

$$
\begin{aligned}
W_{n}(\alpha, s)= & \langle n|T(\alpha, s)| n\rangle \\
= & \frac{2}{1-s}\left(\frac{s+1}{s-1}\right)^{n} \\
& \quad \times \exp \left(-\frac{2|\alpha|^{2}}{1-s}\right) L_{n}\left(\frac{4|\alpha|^{2}}{1-s^{2}}\right) .
\end{aligned}
$$

The function $W_{n}(\alpha, s)$ is analytic in $s$ except for an essential singularity at $s=1$, the pole at $s=-1$ in the polynomial being cancelled by the zero of the factor which multiplies it. By setting $s=1-\epsilon, 0,-1$, we find for the state $|n\rangle$

$$
\begin{aligned}
W_{n}(\alpha, 1) & =\pi P_{n}(\alpha) \\
& =(-1)^{n} e^{|\alpha|^{2}} \frac{d^{n}}{d\left(|\alpha|^{2}\right)^{n}} \delta\left(|\alpha|^{2}\right), \quad(7.25) \\
W(\alpha, 0) & =W_{n}(\alpha)=2(-1)^{n} e^{-2|\alpha|^{2}} L_{n}\left(4|\alpha|^{2}\right),
\end{aligned}
$$


$W_{n}(\alpha,-1)=\left\langle\alpha\left|\rho_{n}\right| \alpha\right\rangle=(n !)^{-1}|\alpha|^{2 n} e^{-|\alpha|^{2}}$,

where $\delta(x)$ is the one-dimensional $\delta$ function.

The two examples considered thus far have had the property that the weight function $P(\alpha)=\pi^{-1} W(\alpha, 1)$ of the $P$ representation exists either as a well-behaved function or as a tempered distribution. This property cannot be true for the pure state that is the superposition ${ }^{26}$ of two coherent states,

$$
|\psi\rangle=N^{-1 / 2}(|\gamma\rangle+|\delta\rangle),
$$

since it is not of the form noted in Sec. III. The normalization factor $N$ is given by

$$
N=2+2 \exp \left(-\frac{1}{2}|\gamma-\delta|^{2}\right) \cos \left[\operatorname{Im}\left(\gamma^{*} \delta\right)\right]
$$

and by using Eq. (I.6.35) we find easily

$$
\begin{aligned}
W(\alpha, s)= & \langle\psi|T(\alpha, s)| \psi\rangle \\
= & \frac{2 N^{-1}}{1-s}\left[\exp \left(-\frac{2|\alpha-\delta|^{2}}{1-s}\right)+\exp \left(-\frac{2|\alpha-\gamma|^{2}}{1-s}\right)\right. \\
& +\langle\gamma \mid \delta\rangle \exp \left(-\frac{2}{1-s}\left(\gamma^{*}-\alpha^{*}\right)(\delta-\alpha)\right) \\
& \left.+\langle\delta \mid \gamma\rangle \exp \left(-\frac{2}{1-s}(\gamma-\alpha)\left(\delta^{*}-\alpha^{*}\right)\right)\right] .
\end{aligned}
$$

For this state $x(\rho)=1$, but because of the second two exponential terms $W(\alpha, s)$ is not a tempered distribution on the line $\operatorname{Re} s=1$.

For this density operator the functions $W(\alpha, 0)$ and $W(\alpha,-1)$ are given by

$$
\begin{aligned}
W(\alpha, 0)=W(\alpha)= & 2 N^{-1}\left(e^{-2|\alpha-\delta|^{2}}+e^{-2|\alpha-\gamma|^{2}}\right. \\
& +\langle\gamma \mid \delta\rangle e^{-2\left(\gamma^{*}-\alpha^{*}\right)(\delta-\alpha)} \\
& \left.+\langle\delta \mid \gamma\rangle e^{-2(\gamma-\alpha)\left(\delta^{*}-\alpha^{*}\right)}\right)
\end{aligned}
$$

and by

$$
\begin{aligned}
W(\alpha,-1)=\langle\alpha|\rho| \alpha\rangle= & N^{-1}\left(e^{-|\alpha-\delta|^{2}}+e^{-|\alpha-\gamma|^{2}}\right. \\
& +\langle\gamma \mid \delta\rangle e^{-\left(\gamma^{*}-\alpha^{*}\right)(\delta-\alpha)} \\
& \left.+\langle\delta \mid \gamma\rangle e^{-(\gamma-\alpha)^{(}\left(\delta^{*}-\alpha^{*}\right)}\right),
\end{aligned}
$$

which are clearly well behaved.

Let us next consider the parametrized family of density operators

$$
\rho\langle n\rangle=\frac{2}{\langle n\rangle+2} \sum_{n=0}^{\infty}\left(\frac{\langle n\rangle}{\langle n\rangle+2}\right)^{n}|2 n\rangle\langle 2 n|,
$$

where $\langle n\rangle$ is the mean number of quanta and only even numbers of quanta are present. If we set

and by

$$
y=\langle n\rangle /(\langle n\rangle+2)
$$

$$
t=-(1+\sqrt{ } y) /(1-\sqrt{ } y) \leq-1,
$$

then, by using Eq. (I.6.22), we may write

$$
\rho\langle n\rangle=(1-t)^{-1}\left[T\left(0, t^{-1}\right)-t T(0, t)\right],
$$

so that we have, on using Eq. (I.6.39),

$$
\begin{aligned}
& W(\alpha, s)= \operatorname{Tr}\left[\rho\left\langle_{n}\right\rangle T(\alpha, s)\right] \\
&=\frac{2}{1-t}\left[\frac{t}{s+t} \exp \left(\frac{2|\alpha|^{2}}{s+t}\right)-\frac{1}{s+t^{-1}}\right. \\
&\left.\times \exp \left(\frac{2|\alpha|^{2}}{s+t^{-1}}\right)\right] .
\end{aligned}
$$

The function $W(\alpha, s)$ has essential singularities at $s=-t>1$ and at $s=-t^{-1}<1$. As the mean number of quanta $\langle n\rangle$ tends to infinity, the parameter

$$
x(\rho)=-t^{-1}
$$

approaches zero. The $P$ representation does not exist for any member of this family of density operators. The Wigner distribution and the function $\langle\alpha|\rho| \alpha\rangle$, on the other hand, are given by

$$
W(\alpha, 0)=W(\alpha)=\frac{2}{1-t}\left(e^{2 t-1|\alpha|^{2}}-t e^{2 t|\alpha|^{2}}\right)
$$

and by

$$
\begin{aligned}
W(\alpha,-1)=\langle\alpha|\rho| \alpha\rangle= & \frac{2}{1-t}\left[\frac{t}{t-1} \exp \left(-\frac{2|\alpha|^{2}}{1-t}\right)\right. \\
& \left.+\frac{1}{1-t^{-1}} \exp \left(-\frac{2|\alpha|^{2}}{1-t^{-1}}\right)\right]
\end{aligned}
$$

respectively.

In Eqs. (2.1) and (2.2), the operators $a$ and $a^{\dagger}$ are defined as linear combinations of the operators $q$ and $p$ with coefficients involving an arbitrary real parameter $\lambda$. Let us alter the scale parameter $\lambda$ to the new value $\lambda^{\prime}$ and consider the new set of operators ${ }^{32}$

and

$$
a^{\prime}=(2 \hbar)^{-1 / 2}\left(\lambda^{\prime} q+i \lambda^{\prime-1} p\right)
$$

$$
a^{\prime \dagger}=(2 \hbar)^{-1 / 2}\left(\lambda^{\prime} q-i \lambda^{\prime-1} p\right),
$$

which are appropriate, for example, to the description of a field amplitude in a medium with a different dielectric constant. These operators have the same algebraic properties as the operators $a$ and $a^{\dagger}$. In particular, the operator $a^{\prime}$ possesses a complete set of eigenstates $|\beta\rangle^{\prime}$ :

$$
a^{\prime}|\beta\rangle^{\prime}=\beta|\beta\rangle^{\prime} \text {. }
$$

We now consider the ground state $|0\rangle^{\prime}$ in the primed system. In terms of the operator

$$
D^{\prime}(\xi) \equiv \exp \left(\xi a^{\prime \dagger}-\xi^{*} a^{\prime}\right),
$$

the characteristic function $\chi^{\prime}(\xi)$ for this density oper-

32 J. R. Klauder, J. McKenna, and D. G. Currie, J. Math. Phys. 6, 734 (1965). 
ator is given by

$$
\begin{aligned}
\chi^{\prime}(\xi) & \equiv\left\langle\left\langle 0\left|D^{\prime}(\xi)\right| 0\right\rangle^{\prime}\right. \\
& =\langle 0 \mid \xi\rangle^{\prime}=e^{-|\xi|^{2} / 2} .
\end{aligned}
$$

By putting $r=\lambda^{-1} \lambda^{\prime}$ and $\xi=x+i y$, we see that

$$
D(x+i y)=D^{\prime}\left(r x+i r^{-1} y\right) \text {. }
$$

Thus we find for the unprimed characteristic function

the value

$$
\chi(\xi) \equiv{ }^{\prime}\langle 0|D(\xi)| 0\rangle^{\prime}
$$

$$
\chi(x, y)=\exp \left[-\frac{1}{2}\left(r^{2} x^{2}+r^{-2} y^{2}\right)\right]
$$

where

$$
\chi(x, y) \equiv \chi(x+i y) .
$$

If we now use Eq. (6.24), we find

$$
W(\alpha, s)=\int \exp \left(\alpha \xi^{*}-\alpha^{*} \xi+\frac{1}{2} s|\xi|^{2}\right) \chi(\xi) \pi^{-1} d^{2} \xi,
$$

or, in real notation with $W(u, v, s) \equiv W(u+i v, s)$,

$$
\begin{aligned}
W(u, v, s)= & \int \exp \left[2 i(x v-y u)+\frac{1}{2}\left(s-r^{2}\right) x^{2}\right. \\
& =\frac{2}{\left[\left(r^{2}-s\right)\left(r^{-2}-s\right)\right]^{1 / 2}} \\
& \quad \times \exp \left[-\frac{2 u^{2}}{r^{-2}-s}-\frac{2 v^{2}}{r^{2}-s}\right] .
\end{aligned}
$$

The function $W(\alpha, s)$ has essential singularities at $s=r^{2}$ and at $s=r^{-2}$. By letting the ratio $r=\lambda^{-1} \lambda^{\prime}$ depart sufficiently far from unity, we may move one of the singularities down to the line $\operatorname{Res}=0$ and make the parameter

arbitrarily small.

$$
x(\rho)=\min \left(r^{2}, r^{-2}\right)
$$

The $P$ representation does not exist for any member of this family of density operators, except for the special cases $r= \pm 1$, for which we have

$$
P(\alpha)=\delta^{(2)}(\alpha) \text {. }
$$

By setting $s=0$ and $s=-1$ in Eq. (7.41), we find that the Wigner function $W(\alpha)$ and the function $\langle\alpha|\rho| \alpha\rangle$ are given by

$$
W(u, v, 0)=2 \exp \left(-2 r^{2} u^{2}-2 r^{-2} v^{2}\right)
$$

and by

$$
W(u, v,-1)=\frac{2}{|r|+|r|^{-1}} \exp \left(\frac{-2 r^{2} u^{2}-2 v^{2}}{r^{2}+1}\right),
$$

respectively.

As a final illustration, let us consider the expectation values of the $s$-ordered products for the state $|0\rangle^{\prime}$.
They are given by the integrals

$$
\begin{aligned}
& { }^{\langle 0|}\left\{\left(a^{\dagger}\right)^{n} a^{m}\right\}_{s}|0\rangle^{\prime} \\
& =(\mu \nu)^{1 / 2} \int(u-i v)^{n}(u+i v)^{m} e^{-\mu u^{2}-\nu v^{2}} \pi^{-1} d u d v,
\end{aligned}
$$

where we have put

$$
\mu^{-1}=\frac{1}{2}\left(r^{-2}-s\right), \quad \nu^{-1}=\frac{1}{2}\left(r^{2}-s\right) .
$$

If we put $n=m$ we may find a generating function for these quantities by forming the series

$$
\begin{aligned}
\sum_{n=0}^{\infty}(n !)^{-1} y^{n} & \left\langle 0\left|\left\{\left(a^{\dagger}\right)^{n} a^{n}\right\}_{s}\right| 0\right\rangle^{\prime} \\
& =(\mu \nu)^{1 / 2} \int \exp \left[-(\mu-y) u^{2}-(\nu-y) v^{2}\right] \pi^{-1} d u d v \\
& =\left(1-\frac{(\mu+\nu) y}{\mu^{2}}+\frac{y^{2}}{\mu \nu}\right)^{1 / 2} .
\end{aligned}
$$

If we now compare this relation with the generating function $^{33}$ for the Legendre polynomials $P_{n}(z)$,

$$
\left(1-2 h z+h^{2}\right)^{-1 / 2}=\sum_{n=0}^{\infty} h^{n} P_{n}(z),
$$

then we find, on making the identifications

$$
h=(\mu \nu)^{-1 / 2} y, \quad z=\frac{1}{2}\left[(\mu / \nu)^{1 / 2}+(\nu / \mu)^{1 / 2}\right],
$$

the result

$$
\begin{aligned}
& { }^{\langle 0|}\left\{\left(a^{\dagger}\right)^{n} a^{n}\right\}_{s}|0\rangle^{\prime} \\
& =n !(\mu \nu)^{-n / 2} P_{n}\left(\frac{1}{2}\left[(\mu / \nu)^{1 / 2}+(\nu / \mu)^{1 / 2}\right]\right) \\
& =n ! 2^{-n}\left[\left(r^{2}-s\right)\left(r^{-2}-s\right)\right]^{-n / 2} \\
& \quad \times P_{n}\left(\frac{1}{2}\left[\left(\frac{r^{2}-s}{r^{-2}-s}\right)^{1 / 2}+\left(\frac{r^{-2}-s}{r^{2}-s}\right)^{1 / 2}\right]\right) .
\end{aligned}
$$

In particular, for $s=0$ we have

$$
{ }^{\prime}\left\langle 0\left|\left\{\left(a^{\dagger}\right)^{n} a^{n}\right\}_{0}\right| 0\right\rangle^{\prime}=n ! 2^{-n} P_{n}\left(\frac{1}{2}\left(r^{2}+r^{-2}\right)\right),
$$

from which the mean number of quanta may be shown to be

$$
\langle n\rangle={ }^{\prime}\left\langle 0\left|a^{\dagger} a\right| 0\right\rangle^{\prime}=\frac{1}{4}\left(r-r^{-1}\right)^{2}=\frac{1}{4}\left(\lambda / \lambda^{\prime}-\lambda^{\prime} / \lambda\right)^{2} .
$$

\section{ANALOGY WITH HEAT DIFFUSION}

It is possible to draw an instructive analogy between the function $W(\alpha, s)$ and the temperature distribution on a plane. We discuss this analogy in the present section and draw from it the conclusion that for every density operator the function $W(\alpha, s)$ must at some point in the half-plane Res $>0$ either assume negative values or cease to be normalized.

${ }^{33}$ Reference 29, p. 51. 
By differentiating the Gaussian convolution (6.32) or, equivalently, by using Eq. (I.7.33), we find that the function $W(\alpha, s)$ obeys the second-order partial differential equation

$$
\frac{\partial W(\alpha, s)}{\partial s}=-\frac{1}{2} \frac{\partial^{2} W(\alpha, s)}{\partial \alpha^{*} \partial \alpha} .
$$

If we express this equation in terms of real coordinates by writing $\alpha=x+i y$ and $W(x, y, s)=W(x+i y, s)$, then it assumes the form

$$
\frac{\partial W(x, y, s)}{\partial s}=-\frac{1}{8}\left(\frac{\partial^{2}}{\partial x^{2}}+\frac{\partial^{2}}{\partial y^{2}}\right) W(x, y, s) .
$$

This is a species of heat-diffusion equation in which the function $W(x, y, s)$ plays the role of the temperature at the point $(x, y)$ and the variable $s$ corresponds in a negative sense to the time variable, $s=-t$. In this analogy the convolution integral (6.32) is Poisson's solution for the temperature in terms of the temperature distribution at an earlier time.

The differential equation (8.2) may be viewed as a conservation law expressing the assumed absence of sources or sinks of heat. It is a well-known feature of solutions to the heat-diffusion equation that, in general, they cannot be extrapolated into the past without the appearance of singularities at some point. These singularities, which represent the sources that originally supplied heat to the plane, correspond to the singularities exhibited by the function $W(\alpha, s)$ for Res $>0$.

We have seen in Eqs. (6.20) and (6.28) that the function $W(\alpha, s)$ is normalized and non-negative when $s$ is real and $\leq-1$. It therefore corresponds to a temperature distribution that for times later than $t=-s=1$ is non-negative and describes a unit amount of heat on the plane. With the passage of time the heat diffuses over the plane according to the convolution integral (6.32). Since there is only a unit amount of heat at time $t=-s=+1$ and since the plane is of infinite extent, the temperature at each point on the plane tends ultimately to zero after a sufficiently long time. This property of temperature distributions is true also of the function $W(\alpha, s)$ since, according to the inequality (6.29), the modulus of $W(\alpha, s)$ goes to zero as $-s=t$ tends to infinity.

It is evident that a temperature distribution that is normalized and non-negative at the time $t=-s=1$ cannot have been normalized and non-negative at all prior times. For if this were the case, then by the argument given in the preceding paragraph the temperature would be zero at all points of the plane. We may prove the corresponding statement for the function $W(\alpha, s)$ by using the convolution law (6.32).

Let us assume that the function $W(\alpha, s)$ is nonnegative and normalized at some real and positive value of $s=u>0$. Then for $\operatorname{Res}<u$, the modulus of the exponential which appears in the integral (6.32), where we replace $t$ by $u$ to avoid confusion with the time variable, is less than unity. We have, therefore, since $W(\beta, u)$ is non-negative by assumption, the inequality

$$
|W(\alpha, s)| \leq\left|\frac{2}{u-s}\right| \int W(\beta, u) \frac{d^{2} \beta}{\pi} .
$$

If the function $W(\beta, u)$ is also normalized in the sense of Eq. (6.20), then the integral over $\beta$ is unity and we may write

$$
|W(\alpha, s)| \leq 2 /|u-s|
$$

for $\operatorname{Res}<u$. Thus if the function $W(\alpha, u)$ were nonnegative and normalized for all positive values of $u$, then, by letting $u$ approach infinity in this inequality, we would find that $W(\alpha, s)$ is identically zero. We have shown, therefore, that the function $W(\alpha, s)$ must either assume negative values or cease to be normalized at some point $s=u>0$.

If the modulus of the function $W(\alpha, s)$ exceeds the value $2|u-s|^{-1}$ specified by Eq. (8.3) for any value of $u>\operatorname{Res}$, then we may conclude that the function $W(\alpha, s)$ either assumes negative values or is not normalized for $s=u$. The smallest value of $u$ for which the inequality (8.3) is violated may therefore be used as an estimate (or, more precisely, an upper bound) for the smallest value of Res at which the function $W(\alpha, s)$ takes on negative values or ceases to be normalized.

We may illustrate the use of this estimate by considering the density operator $T(\gamma, t)$ for which the function $W(\alpha,-1)$ is given by Eq. (7.11) as

$$
W(\alpha,-1)=\frac{1}{\langle n\rangle+1} \exp \left(-\frac{|\alpha-\gamma|^{2}}{\langle n\rangle+1}\right),
$$

where $\langle n\rangle=-\frac{1}{2}(t+1)$ is the mean number of quanta. This function attains a maximum value of $(\langle n\rangle+1)^{-1}$ at $\alpha=\gamma$. Thus by setting $s=-1$ in Eq. (8.3), we find that the inequality

$$
\frac{1}{\langle n\rangle+1} \leq \frac{2}{u+1}
$$

breaks down at

$$
u=1+2\langle n\rangle .
$$

We estimate then that the function $W(\alpha, s)$ will misbehave in some way at this point or at a smaller value of Res. According to Eq. (7.8) the function $W(\alpha, s)$ has an essential singularity at $s=1+2\langle n\rangle$ for which value it may be written as

$$
W(\alpha, 1+2\langle n\rangle)=\pi \delta^{(2)}(\alpha-\gamma) .
$$

This singularity corresponds to a unit amount of heat concentrated at a point $\alpha=\gamma$ at the time $t=-1-2\langle n\rangle$.

\section{TIME-REVERSED HEAT DIFFUSION AND $W(\alpha, s)$}

According to the analogy developed in Sec. VIII, the extension of the function $W(\alpha, s)$ from one value 
of $s$ to another with a larger real part corresponds to the continuation of an initial temperature distribution to prior times. We derive various procedures for doing this in the present section.

One method is to make use of the simple exponential dependence of the function $\chi(\xi, s)$ upon the variable $s$,

$$
\chi(\xi, s)=e^{(s-t)|\xi|^{2} / 2} \chi(\xi, t),
$$

in conjunction with the complex Fourier transform relations (6.23) and (6.24) which connect the functions $W$ and $\chi$. In this way we express the function $W(\alpha, s)$ in terms of $W(\beta, t)$ as the double integral

$$
\begin{aligned}
& W(\alpha, s)=\iint \exp \left[(\alpha-\beta) \xi^{*}-(\alpha-\beta) * \xi\right. \\
&\left.+\frac{1}{2}(s-t)|\xi|^{2}\right] W(\beta, t) \pi^{-2} d^{2} \beta d^{2} \xi,
\end{aligned}
$$

in which the integration over $\beta$ must be done first unless $\operatorname{Res} \leq \mathrm{Re}$, in which case the double integral reduces to the convolution (6.32).

A second method permits us to avoid integrating twice at the expense of introducing another complex variable. Let us define the function $W(\alpha, \beta, s)$ as the integral

$$
W(\alpha, \beta, s)=\int e^{\alpha \xi^{*}+\beta \xi} \chi(\xi, s) \pi^{-1} d^{2} \xi
$$

This function may be shown ${ }^{2}$ to be analytic in all three variables for $\operatorname{Re}<x(\rho)$. The function $W(\alpha, s)$ is $W\left(\alpha,-\alpha^{*}, s\right)$. Let us consider the integral

$2(s-t)^{-1} \int W\left(\alpha+\gamma, \beta+\gamma^{*}, t\right)$

$$
\times \exp \left[-2(s-t)^{-1}|\gamma|^{2}\right] \pi^{-1} d^{2} \gamma
$$

By writing $W$ in terms of $\chi$, we express it as the double integral

$$
\begin{aligned}
2(s-t)^{-1} \int \chi(\xi, t) \exp \left[(\alpha+\gamma) \xi^{*}\right. & +\left(\beta+\gamma^{*}\right) \xi \\
& \left.-2(s-t)^{-1}|\gamma|^{2}\right] \pi^{-2} d^{2} \gamma d^{2} \xi
\end{aligned}
$$

If $\operatorname{Res}>\operatorname{Re} t$, we may use the formula (I.A2) to integrate over the variable $\gamma$, after which it reduces to

$$
\int \chi(\xi, s) e^{\alpha \xi^{*}+\beta \xi} \pi^{-1} d^{2} \xi
$$

which we recognize as the expression (9.2) for $W(\alpha, \beta, s)$. Thus we have the integral formula

$$
\begin{aligned}
W(\alpha, \beta, s)=\frac{2}{s-t} \int W\left(\alpha+\gamma, \beta+\gamma^{*}, t\right) & \\
& \times \exp \left(\frac{-2|\gamma|^{2}}{s-t}\right) \frac{d^{2} \gamma}{\pi}
\end{aligned}
$$

for $\operatorname{Res}>\operatorname{Re} t$, which if we set $\beta=-\alpha^{*}$ becomes

$$
\begin{aligned}
W(\alpha, s)=\frac{2}{s-t} \int W\left(\alpha+\gamma, \gamma^{*}-\alpha^{*}, t\right) & \\
& \times \exp \left(\frac{-2|\gamma|^{2}}{s-t}\right) \frac{d^{2} \gamma}{\pi} .
\end{aligned}
$$

In a similar fashion, by expressing $W\left(\gamma, \gamma^{*}, t\right)$ in terms of $\chi$, we may verify the relation

$$
\begin{aligned}
W(\alpha, \beta, s)=\frac{2}{s-t} \exp \left(\frac{2 \alpha \beta}{t-s}\right) \int W\left(\gamma, \gamma^{*}, t\right) \\
\quad \times \exp \left(\frac{2\left(\alpha \gamma^{*}+\beta \gamma-|\gamma|^{2}\right)}{s-t}\right) \frac{d^{2} \gamma}{\pi}
\end{aligned}
$$

for $\operatorname{Re} s>\operatorname{Ret}$. At $\beta=-\alpha^{*}$ this relation reduces to

$$
\begin{aligned}
W(\alpha, s)=\frac{2}{s-t} \exp \left(\frac{2|\alpha|^{2}}{s-t}\right) \int W\left(\gamma, \gamma^{*}, t\right) \\
\quad \times \exp \left(\frac{2\left(\alpha \gamma^{*}-\alpha^{*} \gamma-|\gamma|^{2}\right)}{s-t}\right) \frac{d^{2} \gamma}{\pi}
\end{aligned}
$$

We may illustrate these relations if we observe that according to Eq. (5.22) the weight function $R\left(\beta^{*}, \alpha\right)$ of the $R$ representation and the function $W(\alpha, \beta, s)$ stand in the relationship

$$
R\left(\beta^{*}, \alpha\right)=e^{\beta^{*} \alpha} W\left(\alpha,-\beta^{*},-1\right) .
$$

Thus by putting $s=+1$ and $t=-1$ in Eqs. (9.4) and (9.6), we may express the weight function of the $P$ representation as the complex Fourier transforms

$$
P(\alpha)=\pi^{-2} e^{-|\alpha|^{2}} \int e^{\alpha \gamma^{*-\alpha^{*} \gamma}} R\left(\alpha^{*}-\gamma^{*}, \alpha+\gamma\right) d^{2} \gamma
$$

and $d^{2,34}$

$$
P(\alpha)=\pi^{-2} e^{|\alpha|^{2}} \int e^{\alpha \gamma^{*}-\alpha^{*} \gamma} R\left(-\gamma^{*}, \gamma\right) d^{2} \gamma
$$

\section{PROPERTIES COMMON TO ALL QUASI- PROBABILITY DISTRIBUTIONS}

In Sec. VI we introduced a parametrized distribution function $W(\alpha, s)$ in terms of which ensemble averages may be expressed as weighted integrals. It is instructive to consider what features of this scheme are necessary ones that must be shared by any scheme for expressing quantum-mechanical ensemble averages as weighted integrals. The present section is a discussion of this point.

Classically, the ensemble average of a quantity $f(\alpha)$ may be written as an integral over a phase-space

\footnotetext{
${ }^{34}$ C. L. Mehta, Phys. Rev. Letters 18, 752 (1967).
} 
probability distribution $p(\alpha)$ in the form

$$
\langle f\rangle=\int f(\alpha) p(\alpha) \pi^{-1} d^{2} \alpha .
$$

Our method for carrying over some of the simplicity of this expression into the quantum theory is represented by the relation (6.7)

$$
\operatorname{Tr}[\rho F]=\int f(\alpha,-s) W(\alpha, s) \pi^{-1} d^{2} \alpha,
$$

in which the functions $f(\alpha,-s)$ and $W(\alpha, s)$ are related to the operators $F$ and $\rho$ by the correspondences $C(s)$ and $C(-s)$, respectively.

Let us now assume that we have an alternative scheme for expressing the quantities $\operatorname{Tr}(\rho F)$ in forms resembling the classical expression (10.1), i.e., that for every density operator $\rho$ and every operator $F$ we have

$$
\operatorname{Tr}[\rho F]=\int f(\alpha) w(\alpha) \pi^{-1} d^{2} \alpha,
$$

where $f(\alpha)$ refers to $F$ and $w(\alpha)$ to $\rho$. The correspondence between $F$ and $f(\alpha)$ implicit in the scheme (10.3) must be linear since the trace $\operatorname{Tr}[\rho F]$ is linear.

Let us denote by $f_{n, m}(\alpha)$ the function associated with the operator $|n\rangle\langle m|$ and by $X(\alpha)$ the operator defined by $f_{n, m}(\alpha)=\langle n|X(\alpha)| m\rangle$. Since the correspondence $F \leftrightarrow f(\alpha)$ is linear, it must associate with every operator $F$

$$
F=\sum_{n, m=0}^{\infty}|n\rangle\langle n|F| m\rangle\langle m|
$$

the function

$$
\begin{aligned}
f(\alpha) & =\sum_{n, m=0}^{\infty}\langle n|F| m\rangle f_{n, m}(\alpha) \\
& =\operatorname{Tr}[F X(\alpha)] .
\end{aligned}
$$

Thus the correspondence implicit in the alternative scheme (10.3) assumes the general form of the correspondence $C(s)$ :

$$
F \underset{s}{\leftrightarrow} f(\alpha,-s)=\operatorname{Tr}[F T(\alpha,-s)] .
$$

If we now substitute the expression (10.4) for the function $f(\alpha)$ into Eq. (10.3), then we find

$$
\operatorname{Tr}(\rho F)=\int \operatorname{Tr}[F X(\alpha)] w(\alpha) \pi^{-1} d^{2} \alpha .
$$

Thus letting $F=|n\rangle\langle m|$, we have for all states $|n\rangle$ and $|m\rangle$ the result

$$
\langle m|\rho| n\rangle=\int\langle m|X(\alpha)| n\rangle w(\alpha) \pi^{-1} d^{2} \alpha,
$$

which implies that

$$
\rho=\int X(\alpha) w(\alpha) \pi^{-1} d^{2} \alpha .
$$

This expansion corresponds to Eq. (6.8).

By again invoking the linearity of the trace in Eq. (10.3) or by referring directly to Eq. (10.7), we see that the correspondence $\rho \leftrightarrow w(\alpha)$ must also be linear and must therefore assume the form

$$
w(\alpha)=\operatorname{Tr}[\rho Y(\alpha)]
$$

for some set of operators $Y(\alpha)$. This relation, which corresponds to Eq. (6.6), in turn implies, by the argument we have given, that Eq. (10.3) is equivalent to the representation

$$
F=\int Y(\alpha) f(\alpha) \pi^{-1} d^{2} \alpha,
$$

which corresponds to Eq. (6.1).

We now observe that from Eqs. (10.7) and (10.8) we have

$$
w(\alpha)=\int w(\beta) \operatorname{Tr}[X(\beta) Y(\alpha)] \pi^{-1} d^{2} \beta
$$

and from Eqs. (10.4) and (10.9),

$$
f(\alpha)=\int f(\beta) \operatorname{Tr}[X(\alpha) Y(\beta)] \pi^{-1} d^{2} \beta .
$$

Since these relations are assumed to hold for all $\rho$ and $F$, we must have

$$
\operatorname{Tr}[X(\beta) Y(\alpha)]=\pi \delta^{(2)}(\alpha-\beta),
$$

which is the analog of the orthogonality rule

$$
\operatorname{Tr}[T(\alpha, s) T(\beta,-s)]=\pi \delta^{(2)}(\alpha-\beta) .
$$

So far we have exploited only the linearity of the scheme (10.3). If we now assume that it offers some of the simplicity of the classical procedure (10.1) then for some species of ordered product, let us write it as $\left[\left(a^{\dagger}\right)^{n} a^{m}\right]_{q}$, where $q$ denotes the type of ordering, we must have ${ }^{35}$

$$
\operatorname{Tr}\left\{\rho\left[\left(a^{\dagger}\right)^{n} a^{m}\right]_{q}\right\}=\int\left(\alpha^{*}\right)^{n} \alpha^{m} w(\alpha) \pi^{-1} d^{2} \alpha .
$$

It is the type of ordering involved in this relation that distinguishes the arbitrary scheme (10.3) from the procedure of Eq. (6.7). There is a great deal of latitude

${ }^{35}$ The characteristic function $\chi(\alpha)$ and the displacement operators $D(\alpha)$, discussed in Sec. III of $\mathrm{I}$, form the basis for a scheme of the form of Eq. (10.3). The roles of the function $w(\alpha)$ and of the operators $X(\alpha)$ and $Y(\alpha)$ are played by $\chi(\alpha), D(-\alpha)$, and $D(\alpha)$, respectively. Equations $(10.3),(10.4),(10.7)-(10.9)$, and (10.12) correspond to Eqs. (I.3.23), (4.2)-(4.4), (4.1), and (I.3.11), respectively. This scheme does not, however, conform to Eq. (10.13) as is shown, for example, by Eq. (6.26). 
here; for, although we have considered a complex plane of orderings in the procedure of Sec. VI, there exists a considerable variety of other types of ordering. The three ways of ordering the operators $p$ and $q$ considered in Sec. IV of I, for example, are easily generalized to a complex plane of $q, p$ orderings.

If, however, the relation (10.13) should apply to the $s$-ordered products for some value of $s$, then, by forming the ensemble average of the series $(2.7)$ for $D(\xi, s)$, we would obtain the relation

$$
\chi(\xi, s)=\int e^{\xi \alpha^{*}-\alpha^{*} \xi_{w}}(\alpha) \pi^{-1} d^{2} \alpha,
$$

which upon Fourier inversion would imply $w(\alpha)=W(\alpha, s)$.

\title{
Nuclear Interactions and Cosmic Radiation at Energies around $10^{6} \mathrm{GeV}$
}

\author{
C. B. A. McCusker, L. S. Peak, and M. H. Rathgeber \\ Cornell-Sydney University A stronomy Center, The University of Sydney, Sydney, N. S. W., Australia
}

(Received 3 June 1968)

\begin{abstract}
The results of a series of Monte Carlo simulations of extensive air showers are compared with experimental data from the Sydney 64-scintillator array and other experiments. The work has had two main objectives: (a) the study of the composition of cosmic radiation around $10^{6} \mathrm{GeV}$; (b) the study of nuclear interactions at very high energies. In the first field, evidence is produced to show that the composition of cosmic radiation is much the same at $10^{15} \mathrm{eV}$ total energy as at $5 \times 10^{10} \mathrm{eV}$ total energy. Above about $3 \times 10^{15}$ $\mathrm{eV}$ the composition changes, the beam becoming progressively richer in heavier nuclei up to energies of about $10^{17} \mathrm{eV}$. In the second field, the main result reported is that at energies above $10^{14} \mathrm{eV}$ there is evidence that much higher transverse momenta occur than at machine energies. If true, this implies that a force is acting which is much stronger than the normal strong interaction.
\end{abstract}

\section{INTRODUCTION}

$V^{1}$ ERY high-energy nuclear interactions can only be studied using the cosmic radiation. Up to energies of $\sim 2 \times 10^{14} \mathrm{eV}$, occasional interactions in very large emulsion stacks can be found. Above that energy the events become too rare for this method, and then airshower techniques (sometimes involving emulsions) must be used. Several recent experiments ${ }^{1}$ have suggested that new fundamental processes may become important above $10^{14} \mathrm{eV}$; hence it is more important than ever to study this region. In particular, we wish to know the composition of the radiation and how it varies with energy, both because this would make our study of the fundamental interactions easier and also because it is of considerable interest to astrophysicists. In recent years considerable progress has been made experimentally. We can now study the core region of air showers in considerable detail. Until recently, however, progress was hindered because the mathematical methods available were not able to relate these detailed properties of the fundamental parameters of the basic nuclear reactions and the nature of the primary particle. However, this is no longer so. With the improvement in speed and storage capacity of modern computers it has

* Work supported by the Science Foundation for Physics of the University of Sydney, and by the U. S. Air Force Office of Scientific Research under Grant No. AF-AFOSR-676-66.

${ }^{1}$ C. B. A. McCusker, Can. J. Phys. 46, 397 (1968). become possible to calculate quite fine details in airshower core structure and so on from the fundamental parameters using Monte Carlo techniques. In this paper we report the results of such calculations using four different primary particles (with $A=1,4,16$, and 64 ), eight different models of the fundamental nuclear interaction, and three different primary energies. These theoretical calculations are then compared with the results of experiments at Sydney and elsewhere.

We conclude that there is good evidence for the occurrence of very high transverse momenta in nuclear interactions at energies $\gtrsim 10^{14} \mathrm{eV}$ and that this implies the existence of some very strong force; that the composition of the cosmic ray beam is roughly constant up to about $2 \times 10^{15} \mathrm{eV}$; and that from this energy up to about $10^{17} \mathrm{eV}$ it becomes progressively richer in heavier nuclei.

\section{SIMULATION PROCESS}

In the simulation process we supposed that incident particles of atomic weight $A$ and energy $E_{p}$ were incident vertically on an exponential atmosphere and aimed at the center of a $9 \times 9$ array of scintillators. Each scintillator measured $0.5 \times 0.5 \mathrm{~m}$, and they were in contact. The position in the atmosphere of each interaction of all hadrons was sampled by the Monte Carlo technique from a distribution giving a mean free path of $\lambda \mathrm{g} / \mathrm{cm}^{2}$. The numerical value of $\lambda$ was $90 \mathrm{~g} / \mathrm{cm}^{2}$ for protons and pions, and 65,43 , and $42 \mathrm{~g} / \mathrm{cm}^{2}$, respec- 